\title{
Parallel Genome-Wide Expression Profiling of Host and Pathogen During Soybean Cyst Nematode Infection of Soybean
}

\author{
Nagabhushana Ithal, ${ }^{1}$ Justin Recknor, ${ }^{2}$ Dan Nettleton, ${ }^{2}$ Leonard Hearne, ${ }^{3}$ Tom Maier, ${ }^{4}$ \\ Thomas J. Baum, ${ }^{4}$ and Melissa G. Mitchum ${ }^{1}$ \\ ${ }^{1}$ Division of Plant Sciences, University of Missouri, Columbia 65211, U.S.A.; ${ }^{2}$ Department of Statistics, lowa State \\ University, Ames 50011, U.S.A.; ${ }^{3}$ Department of Statistics, University of Missouri, Columbia 65211, U.S.A.; ${ }^{4}$ Department \\ of Plant Pathology, lowa State University, Ames 50011, U.S.A.
}

Submitted 5 April 2006. Accepted 10 October 2006.

\begin{abstract}
Global analysis of gene expression changes in soybean (Glycine max) and Heterodera glycines (soybean cyst nematode [SCN]) during the course of infection in a compatible interaction was performed using the Affymetrix GeneChip soybean genome array. Among 35,611 soybean transcripts monitored, we identified 429 genes that showed statistically significant differential expression between uninfected and nematode-infected root tissues. These included genes encoding enzymes involved in primary metabolism; biosynthesis of phenolic compounds, lignin, and flavonoids; genes related to stress and defense responses; cell wall modification; cellular signaling; and transcriptional regulation. Among 7,431 SCN transcripts monitored, 1,850 genes showed statistically significant differential expression across different stages of nematode parasitism and development. Differentially expressed SCN genes were grouped into nine different clusters based on their expression profiles during parasitism of soybean roots. The patterns of gene expression we observed in SCN suggest coordinated regulation of genes involved in parasitism. Quantitative real-time reverse-transcription polymerase chain reaction confirmed the results of our microarray analysis. The simultaneous genome-wide analysis of gene expression changes in the host and pathogen during a compatible interaction provides new insights into soybean responses to nematode infection and the first profile of transcript abundance changes occurring in the nematode as it infects and establishes a permanent feeding site within a host plant root.
\end{abstract}

Additional keywords: esophageal gland cell, parasitism genes, syncytium.

The soybean cyst nematode ( $\mathrm{SCN}$ ), Heterodera glycines, is an obligate, sedentary endoparasite which is consistently the most damaging pest of soybean (Wrather et al. 2001). The nematode life cycle consists of five life stages punctuated by four molts. After the first molt within the egg, SCN secondstage juveniles (J2) hatch into the soil and are attracted to host

Corresponding author: Melissa G. Mitchum; Telephone: 573-882-6152; Fax: 573-884-9676; E-mail: goellnerm@missouri.edu

* The $\boldsymbol{e}$-Xtra logo stands for "electronic extra" and indicates the HTML abstract available on-line contains supplemental material not included in the print edition. Three supplemental tables appear on-line. plant roots by diffusates. Within hours of arriving at the root surface, the nematode has penetrated through epidermal cells and migrated intracellularly to the vascular cylinder of the root. Once the migratory juvenile reaches the vasculature, it selects an initial syncytial cell to initiate a permanent feeding site called a syncytium. The syncytium supplies the nutritional needs of the parasitic nematode to complete its life cycle (Endo 1986; Hussey and Grundler 1998). Once the juvenile begins to feed from the syncytium, it becomes sedentary and undergoes three more molts to either an adult vermiform male or a pyriform female. The male migrates back out of the root to fertilize the lemon-shaped female, which protrudes from the root, and then dies. The adult female secretes some of her eggs in a gelatinous matrix outside her body. However, the majority of eggs are retained within the uterus. Once the female dies, her body serves as a cyst to protect the eggs in the soil. Under favorable environmental conditions, an entire life cycle is completed in 25 to 30 days. The growth and development of the nematode is dependent on the successful establishment of a feeding cell, such that any impairment in syncytium formation can delay or completely disrupt further development.

Several studies have demonstrated changes in host gene expression during nematode infection of roots, specifically within the developing syncytium (Gheysen and Fenoll 2002; Hussey et al. 2002; Puthoff et al. 2003; Williamson and Gleason 2003). Evidence suggests that the process of syncytium formation involves alterations in basic aspects of programmed plant development, where normal cells dedifferentiate into a highly metabolically active cell type involving alterations to the cell cycle, hormone regulation, and cell wall architecture (Bird 1996; Bird and Koltai 2000; Davis and Mitchum 2005; Goellner et al. 2001; Hussey and Grundler 1998). Some of the processes leading to the establishment of nematode feeding cells are believed to be initiated and modulated by the encoded products of nematode parasitism genes expressed in the esophageal glands and delivered via the nematode's stylet directly into or within the vicinity of the initial feeding cell (Davis et al. 2000, 2004).

Different approaches have been employed to study the plant response to cyst nematode parasitism, including differential display (Hermsmeier et al. 1998, 2000; Vercauteren et al. 2001), promoter-reporter gene fusions (Barthels et al. 1997; Favery et al. 1998; Goddijn et al. 1993; Goverse et al. 1998; Mazarei et al. 2003, 2004; Niebel et al. 1996; Puzio et al. 2000), RNA blotting, protein immunolocalization, in situ hybridization (Goellner et al. 2001; Niebel et al. 1993), and differential library screening (Gurr et al. 1991). Similarly, con- 
struction of cDNA libraries using microaspirated contents of the esophageal gland cells of SCN parasitic stages has led to identification of several genes that may play critical roles in parasitism (Gao et al. 2001, 2003; Wang et al. 2001). Although these approaches have yielded valuable insights into the host response and nematode parasitism, the total number of identified cyst nematode and host plant genes involved in this hostparasite interaction still is relatively small.

Oligonucleotide and cDNA microarrays also have been used to study changes in plant gene expression at a single timepoint during early stages of cyst nematode infection in Arabidopsis (Puthoff et al. 2003) and soybean (Khan et al. 2004). A cDNA microarray also was used for developmental expression analysis of 1,358 cDNAs from the esophageal gland cell region of SCN (De Boer et al. 2002b). To our knowledge, a microarray study to simultaneously analyze changes in both plant and cyst nematode gene expression during nematode infection and development in host plant roots has not been conducted. Moy and associates (2004) demonstrated the utility of this approach for analyzing host and pathogen gene expression in the compatible soybean-Phytophthora spp. interaction using cDNA microarrays. Availability of cDNA libraries and extensive expressed sequence tag (EST) sequencing efforts in both soybean and SCN have led to the recent development of the GeneChip soybean genome array from Affymetrix, representing 35,611 soybean transcripts and 7,431 SCN transcripts, providing an excellent tool for simultaneous analysis of gene expression changes in both soybean and SCN.

In this study, three timepoints representing different stages of nematode parasitism and development in soybean were chosen for analysis. Our study of the soybean-SCN interaction transcriptome identified a large number of differentially expressed genes in both the host and pathogen. The lack of functional genomics resources in soybean and SCN is a major bottleneck in interpreting the large-scale data generated through the microarray approach. However, earlier studies suggest that homologous soybean and Arabidopsis genes share responsiveness to cyst nematode infection (Mazarei et al. 2003, 2004).
Thus, annotation of soybean genes based on sequence similarity to Arabidopsis genes with known function is useful for aiding in the identification of key biological processes altered during nematode infection. Using this approach, we were able to annotate 320 of the 429 differentially regulated genes to identify alterations in plant gene expression occurring globally over a time course of infection. The observed patterns of SCN gene expression suggest coordinated regulation of genes involved in parasitism, including cell-wall-modifying enzymes (e.g., cellulases and pectinases) and candidate parasitism genes (Gao et al. 2001, 2003; Wang et al. 2001).

\section{RESULTS}

To study the patterns of gene expression changes simultaneously in both the host and pathogen during nematode parasitism in a compatible interaction, we conducted a microarray experiment using Affymetrix GeneChip soybean genome arrays representing probes from both the soybean and SCN genomes. Sampling times at 2, 5, and 10 days postinoculation (dpi) were selected to cover different stages of nematode parasitism. At 2 dpi, the majority of infective $\mathbf{J} 2$ (now parasitic J2 [pJ2]) had arrived at the vascular cylinder and selected an initial syncytial cell to begin feeding (Fig. 1A and 1D). By 5 dpi, the sedentary $\mathrm{pJ} 2$ body was slightly swollen from feeding, had lost its somatic musculature, and may have had undergone the second molt to the early J3 life stage (Fig. 1B). At this timepoint, several cells have coalesced to form the syncytium (Fig. 1E). By $10 \mathrm{dpi}$, the syncytia were well developed and nematodes had advanced to early J4 life stages (Fig. 1C and 1F). Infection experiments were conducted in triplicate, with each experiment consisting of two treatments, mock and SCN-infected. To enrich tissues of interest, whole soybean root segments representing the major zone of infection were excised at different timepoints after inoculation and compared with uninfected excised root segments of the same developmental stage. To reduce variability inherent to the system, each root was inoculated with a known number of sterile infective $\mathrm{J} 2$ and the infection

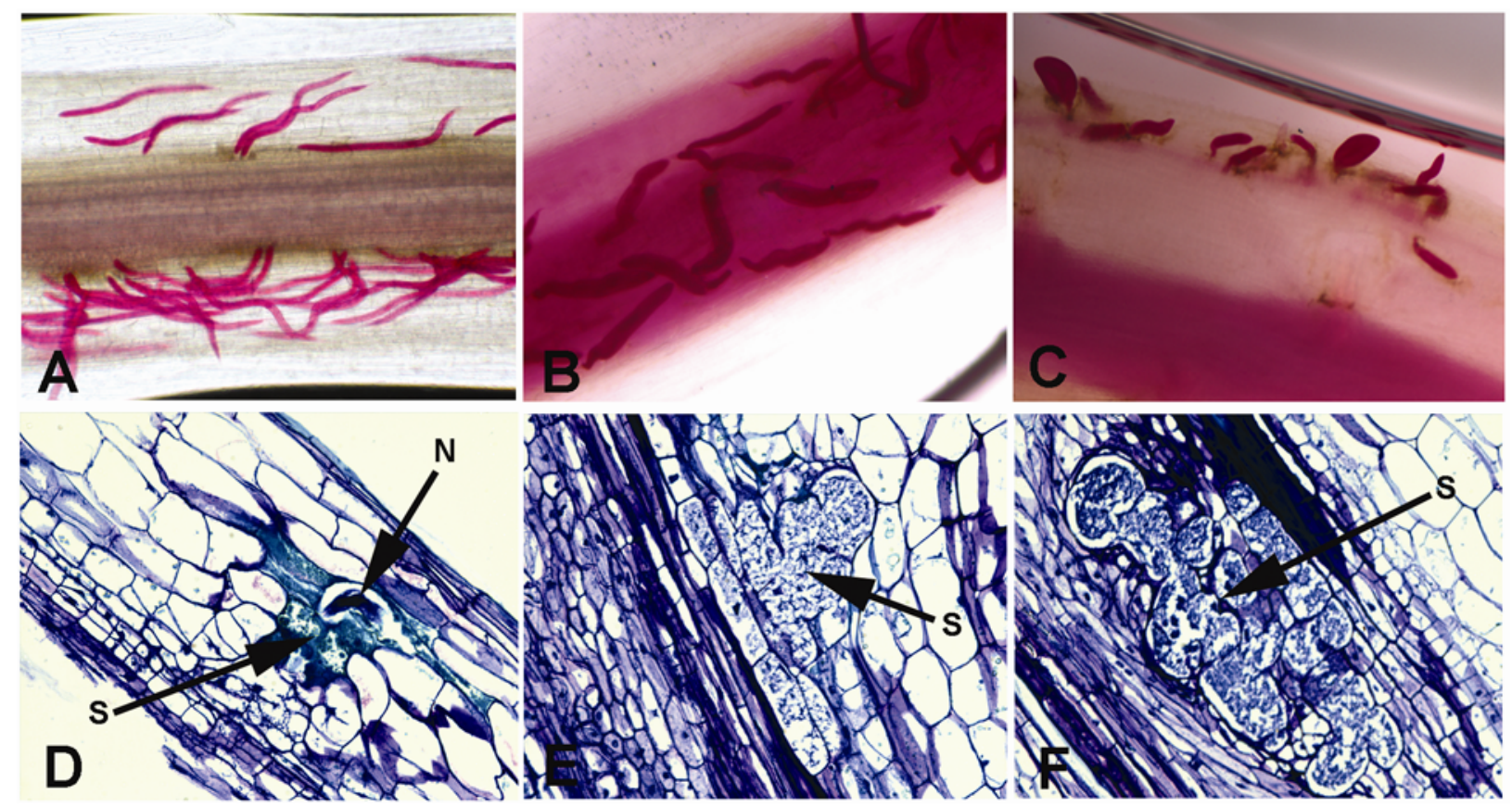

Fig. 1. Developmental stages of soybean cyst nematode race 3 (HG type 0) and syncytia in soybean roots of susceptible cv. Williams 82 . A, Early parasitic second-stage juveniles (J2s) at 2 days postinoculation (dpi), B, Late parasitic J2s and early J3s at 5 dpi. C, Late parasitic J3s and early J4s at 10 dpi. Soybean root sections showing syncytia at $\mathbf{D}, 2 \mathrm{dpi}, \mathbf{E}, 5 \mathrm{dpi}$, and F, $10 \mathrm{dpi}$. Nematodes in whole roots were stained with acid fuschin stain. Soybean root sections were stained with toluidine blue. $\mathrm{N}=$ nematode and $\mathrm{S}=$ syncytium. 
process was synchronized by washing the roots $24 \mathrm{~h}$ after the inoculation to remove any juveniles that had not yet penetrated the tissue. Furthermore, at least 30 root segments containing the sedentary nematodes were harvested and bulked for each timepoint. To avoid the early migratory phase of the infection process and the gene expression changes typically associated with this phase of infection, a relatively late initial timepoint of 2 dpi was selected because, at that time, most juveniles had reached the vasculature of the root and initiated feeding.

Statistical analysis was performed separately on soybean and nematode gene expression data derived from the chip analyses. Data from both SCN-infected and mock treatments were used to analyze changes in soybean transcript levels. Data from the SCN-infected treatments were used exclusively for the analysis of nematode gene expression.

\section{Soybean gene expression changes during infection.}

This experiment followed a split-plot design (Kuehl 2000) with two fixed factors: treatment (infected or uninfected) and time $(2,5$, or $10 \mathrm{dpi})$. For each soybean gene, we conducted a mixed linear model analysis (described below). As part of each mixed linear model analysis, estimates of mean expression on the log scale were obtained for each combination of treatment and time. Each mixed linear model analysis included a test for treatment main effects and a test for treatment-time interaction. The test for treatment main effects tests the null hypothesis of no difference between treatments when averaging over time according to the equation $\mathrm{H}_{0}:\left(\mathrm{I}_{2}+\mathrm{I}_{5}+\mathrm{I}_{10}\right) / 3=\left(\mathrm{U}_{2}+\mathrm{U}_{5}+\right.$ $\left.\mathrm{U}_{10}\right) / 3$, where $\mathrm{I}_{2}, \mathrm{I}_{5}, \mathrm{I}_{10}, \mathrm{U}_{2}, \mathrm{U}_{2}$, and $\mathrm{U}_{10}$ denote the means corresponding to the six combinations of treatment and time. The test for interaction tests the null hypothesis $\mathrm{H}_{0}: \mathrm{I}_{2}-\mathrm{U}_{2}=\mathrm{I}_{5}-\mathrm{U}_{5}=$ $\mathrm{I}_{10}-\mathrm{U}_{10}$, which says that the difference between treatment means at each timepoint is equal to a single value common to all timepoints. When controlling the false discovery rate (FDR) at approximately 5\%, 618 soybean genes were found to exhibit significant treatment main effects, and no genes were found to exhibit significant interaction. In the absence of interaction, it suffices to average over time and to consider only treatment main effects. However, because some genes exhibited significant interaction at higher FDRs (data not shown), we also conducted separate tests for treatment effects at each timepoint and have provided those results for the 618 differentially expressed genes online in Supplemental Table 1. These time-specific tests are less powerful than the tests for treatment main effects; thus, there are many genes for which none of the time-specific differences were significant despite the significant treatment main effect. The list of 618 statistically significant genes was further filtered by applying a cutoff of a 1.5fold change between mock and treatment group. The 429 genes exhibiting more than a 1.5 -fold change between at least one of the three treatment groups (mock to treatment at 2, 5, or 10 dpi) were considered biologically significant in this analysis. All the tables in this manuscript are coded by highlighting the fold-change values in dark gray for genes that are upregulated $(\mathrm{q}=0.05$ and $>1.5$-fold change), light gray for genes that are downregulated $(q=0.05$ and $>1.5$-fold change $)$, and white for genes that show only statistically significant $(q=0.05)$ differential expression but did not pass the 1.5 -fold change cutoff.

Very limited functional information is available for the soybean sequences represented on the Affymetrix GeneChip soybean genome array; therefore, we tried to annotate the differentially expressed soybean transcripts by finding orthologs in Arabidopsis. By searching the TAIR Arabidopsis sequence database using the soybean EST sequences as a query, we were able to assign putative functions for 320 of the 429 differentially expressed soybean genes (Supplemental Table 1). Although the putative functions are based solely on nucleotide or amino acid similarity and not experimental evidence, the analysis is useful for selecting candidate genes of interest for further work to obtain biologically relevant functional data. A large proportion of differentially expressed genes appear to be involved in basic plant metabolism. Transcripts for genes involved in plant secondary metabolism, including the biosynthesis of phenolic compounds, lignin, and flavonoids, also were upregulated early during nematode infection and remained elevated throughout nematode development. Similarly, genes for proteins related to stress and defense responses such as pathogenesis-related (PR) proteins, disease resistance responsive proteins, polygalacturonase-inhibiting proteins, harpininduced family proteins, glutathione- $S$-transferases, peroxidases, and osmotin-like proteins were consistently upregulated after infection at all three timepoints. Subsets of cell-wallmodifying enzymes, including expansins, pectinesterases, xyloglucan endotransglycosylase, and extensins, were either upor downregulated. However, the majority of these genes were upregulated during nematode infection. Genes possibly involved in plant growth and development, such as the auxin transport protein gene PIN2 (EIRI), and No Apical Meristem (NAM) also were induced, as were ferritins, iron-binding proteins with a role in maintaining iron homeostasis in plants. Genes involved in cellular signaling and transcriptional activation, such as those encoding bZIP, ERF, MYB, WRKY, and zinc-finger transcription factors, were upregulated. A zinc-finger family transcription factor also was downregulated. Several leucinerich repeat receptor-like kinases (LRR-RLKs) were both upand downregulated.

Cluster analysis was performed on the 429 differentially expressed soybean genes based on a shared pattern of differential expression across adjacent timepoints. Separate clustering was done for uninfected (mock) and SCN-infected treatment groups. Based on this gene clustering method, genes could be classified into nine different clusters for each treatment group. The expression profile of each gene is summarized by a uniquely identifiable cluster combination corresponding to the expression pattern in the uninfected and infected roots. Following Vanneste and associates (2005), we presented the results of this clustering in a cross-table format (Fig. 2A). A prototypical expression pattern is depicted at the heading of each row and column in Figure 2A to facilitate interpretation of the counts in the table. The functional relevance of all cluster combinations was evaluated to gain further insight into the dataset. Mock cluster 5-infected cluster 5 is the largest cluster combination, with 75 genes. The expression of genes in this cluster combination do not exhibit any significant change between adjacent timepoints in either mock or infected roots. However, these genes were significantly differentially expressed between mock and infected roots. The majority of genes in this cluster combination are involved in metabolic processes. All genes with an annotated function in the cluster combination mock cluster 5-infected cluster 2 appear to be involved in defense response. Genes in this cluster combination do not show any significant change in their expression between adjacent timepoints in the mock group but are significantly upregulated from 2 to 5 dpi and stay constant between 5 and 10 dpi in infected roots. Although factors like redundancy on the array and the cross-hybridization of gene families should be taken into consideration when interpreting these results, this list provides a good estimate of the global changes in soybean gene expression during nematode parasitism of roots.

\section{Quantitative real-time reverse-transcription polymerase chain reaction validation of soybean microarray results.}

Quantitative real-time reverse-transcription polymerase chain reaction (qPCR) was used to validate the microarray results. 


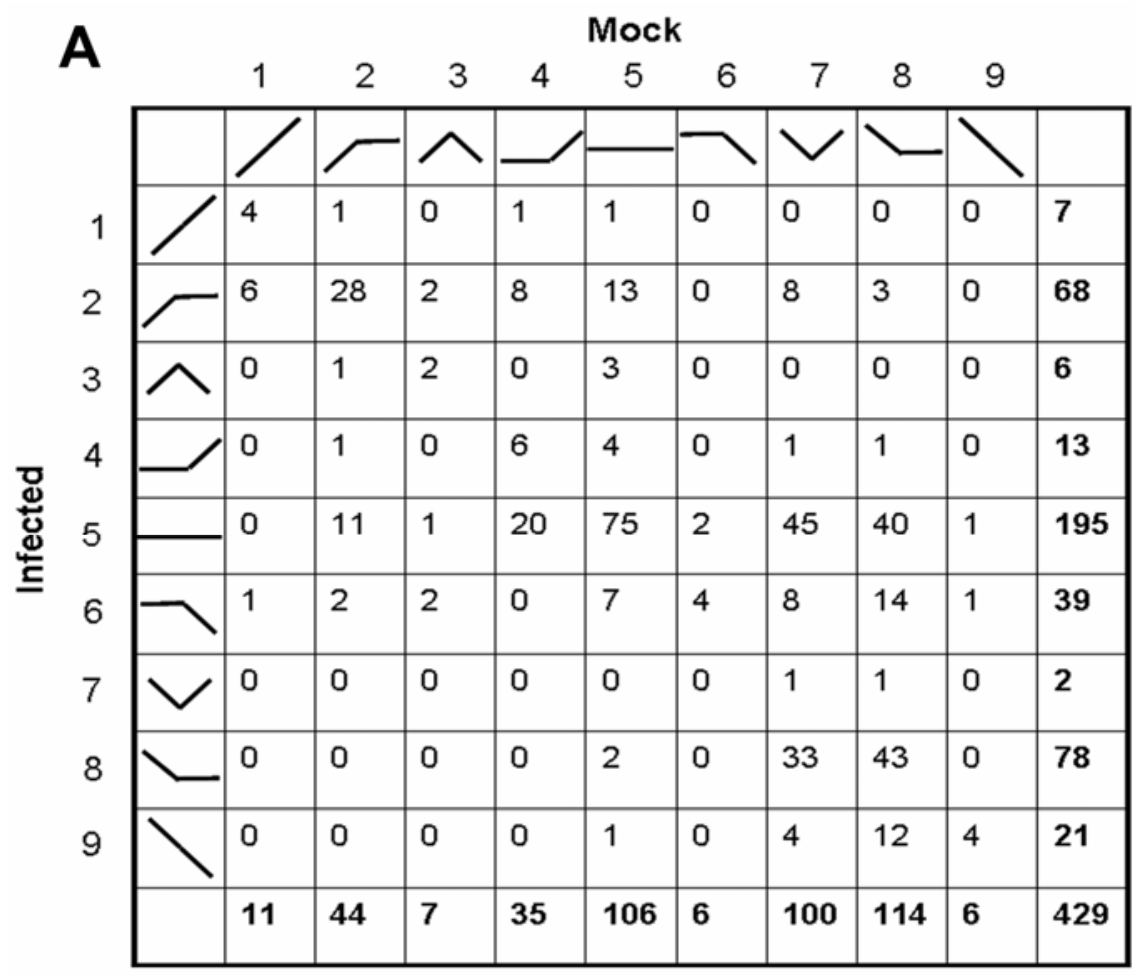

B

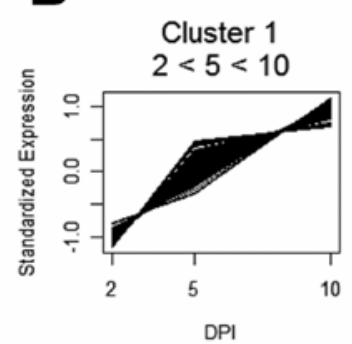

Cluster 4

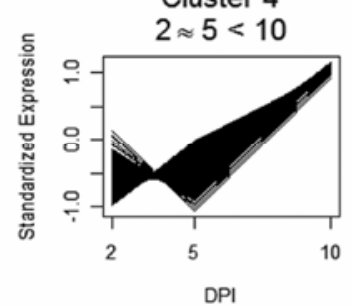

DPI

Cluster 7

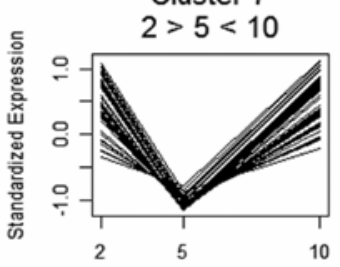

DPI

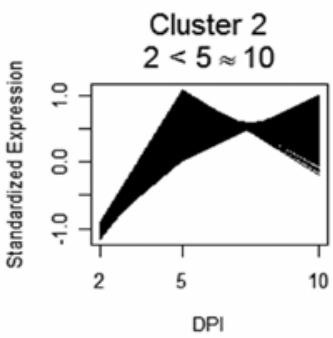

Cluster 5

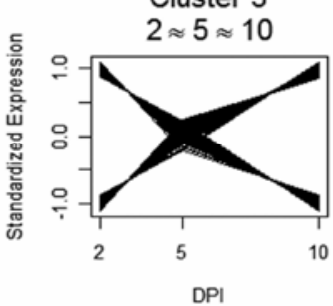

DPI

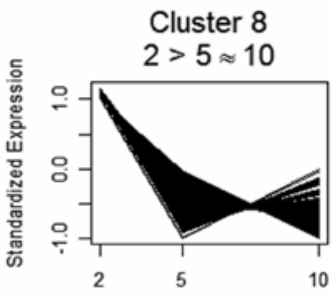

DPI
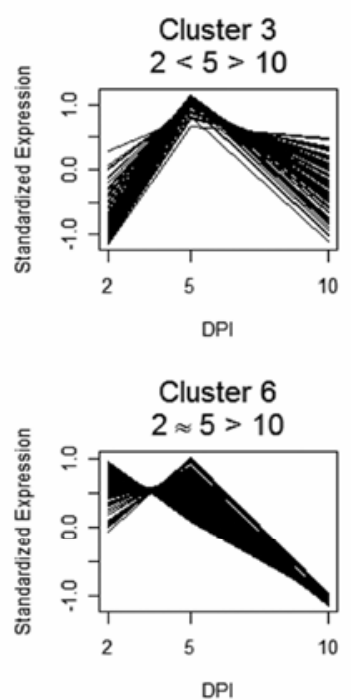

DPI

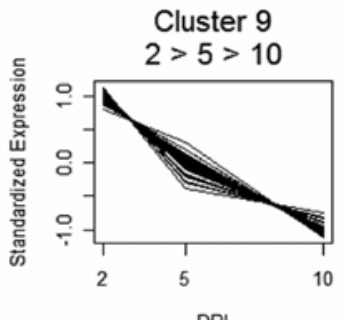

DPI

Fig. 2. A, Cross-table representation of the expression profiles within mock and treatment groups for the 429 differentially expressed soybean genes. The frequencies of each cluster combination within the dataset are indicated in each square. Lines in top and left squares show trend lines for changes in expression pattern across three timepoints. Numbers on the top row and left column indicate the cluster numbers. B, Cluster analysis of differentially expressed soybean cyst nematode ( $\mathrm{SCN})$ genes. Genes are classified into nine different clusters based on the significance of their expression changes across adjacent timepoints. The patterns represent early (clusters 2 and 8), late (clusters 4 and 6), transient (clusters 3 and 7), and continuous (clusters 1 and 9) changes during SCN development. Cluster 5 includes transcripts that exhibited significantly nonconstant expression across the three timepoints but with no significant changes in expression between adjacent timepoints. The genes in this cluster exhibit more gradual increases or decreases in expression than the genes with similar patterns in clusters 1 and 9. For visualization purposes, each gene's estimated mean log-scale expression profile was standardized to have mean 0 and variance 1 prior to plotting. 
The 19 genes and primers used for qPCR validation to represent different expression patterns and their putative functions are listed in Supplemental Table 2. Total RNA from the same biological replicates used for microarray analysis was used for qPCR. These experiments included triplicate technical replication for each biological replicate. The quantification of gene expression was performed using the relative quantification $\left(\Delta \Delta \mathrm{C}_{\mathrm{T}}\right)$ method and comparing the data with internal controls. The quantitative PCR results, by and large, are in agreement with DNA array hybridization results (Table 1). The estimated direction of the treatment effect was consistent for 49 of the 57 comparisons (approximately 86\%), and the correlation between the $\log _{2}$ fold changes estimated by microarray and qPCR was 0.863 . If we average over time, as was done in our microarray analysis due to the absence of significant interaction, the agreement between the microarray and qPCR results improves. The estimated direction of the treatment main effect was identical for all genes examined, and the correlation between the $\log _{2}$ fold changes averaged over time was 0.887 . The differences between qPCR and microarray results include genes encoding an ethylene response factor $(E R F)$, LeMir homolog, chalcone synthase, polygalacturonase-inhibiting protein $1(P G I P-1)$, and PR protein $1(P R l)$, which all were found to be upregulated in our microarray analysis at 2 dpi but were slightly downregulated in qPCR analysis. However, the microarray and qPCR results were qualitatively in agreement for these genes at later timepoints. We also observed differences for a gene encoding an expansin family protein which was found to be downregulated in microarray analysis at $2 \mathrm{dpi}$ but shown to be upregulated by qPCR. Observed differences between microarray and qPCR were confined to the 2 dpi timepoint, with the exception of genes encoding a MATE efflux family protein and a cytochrome P450 which showed differences only at $10 \mathrm{dpi}$. This is likely due to the high level of variability inherent to the $2 \mathrm{dpi}$ timepoint as a result of the migratory phase of the nematode life cycle, inability to control the number of nematodes in the roots, and the small size of syncytia. Differences in the magnitude of expression for several genes as detected by the two different methods also were observed (Table 1). The differences between the microarray results and some of the qPCR results are likely attributed to the differences in sensitivity of the two different approaches, possibly due to cross hybridization of closely related gene families in microarray experiments, as well as the variability associated with the inability to control the number of nematodes within the root, which affects the number of host cells responding to infection in separate experiments.

\section{Expression profile of nematode genes during infection.}

Nematode gene expression data from the SCN-infected treatments at three different timepoints were compared because there were no nematode transcripts present in mock-infected tissues for pairwise comparison. Thus, nematode genes identified as differentially expressed across these timepoints are the genes that are induced or suppressed during nematode infection and development. In total, 1,871 genes were identified as differentially expressed during nematode development at a 5\% FDR, of which 1,850 genes exhibited a 1.5 -fold or greater difference in expression between at least one of the three time combinations used ( 2 to 5,5 to 10 , or 2 to $10 \mathrm{dpi}$ ). These 1,850 genes were grouped into nine clusters based on changes in their expression across the three timepoints (Fig. 2B). The first row of Figure 2B contains genes whose expression at 2 dpi was significantly less than at 5 dpi. The second row contains genes whose expression levels at 2 and 5 dpi did not differ significantly. The third row contains genes whose expression at 2 dpi was significantly greater than at $5 \mathrm{dpi}$. A list of all differentially expressed nematode genes with their predicted functions and the cluster information is available online in Supplemental Table 3.

Clusters 1,2, and 4 include transcripts that exhibit a significantly increasing pattern of expression over the course of infection. Most of the transcripts in these clusters appear to be involved in nematode development and metabolism. Members of clusters 6,8 , and 9 show a significantly decreasing pattern of expression over the course of infection. The majority of previously identified parasitism gene candidates that are expressed in the nematode esophageal gland cells and encode products containing a signal peptide fall into these three clusters (Table 2). Cluster 3 represents transcripts that were significantly

Table 1. Soybean microarray data validation by quantitative real-time reverse transcription-polymerase chain reaction (qPCR)

\begin{tabular}{|c|c|c|c|c|c|c|c|c|}
\hline \multirow[b]{2}{*}{ Affymetrix probe ID } & \multirow[b]{2}{*}{$\begin{array}{l}\text { GenBank } \\
\text { accession }\end{array}$} & \multirow[b]{2}{*}{ Putative Function } & \multicolumn{6}{|c|}{ Fold change $^{a}$} \\
\hline & & & $\begin{array}{c}2 \text { dpi } \\
\text { micro- } \\
\text { array }\end{array}$ & $\begin{array}{l}\text { qRT- } \\
\text { PCR }\end{array}$ & $\begin{array}{c}5 \text { dpi } \\
\text { micro } \\
\text { array }\end{array}$ & $\begin{array}{l}\text { qRT- } \\
\text { PCR }\end{array}$ & $\begin{array}{l}10 \text { dpi } \\
\text { micro } \\
\text { array }\end{array}$ & $\begin{array}{l}\text { qRT- } \\
\text { PCR }\end{array}$ \\
\hline GmaAffx.47493.1.S1_at & AW307476 & Auxin transport protein (EIR1) PIN2 & 1.32 & 1.21 & 6.02 & 6.97 & 2.38 & 3.21 \\
\hline Gma.12330.2.S1_at & BI972758 & $\begin{array}{l}\text { ERF (ethylene response factor)/AP2 transcription factor } \\
\text { family protein. }\end{array}$ & 1.70 & -1.47 & 2.31 & 2.79 & 1.89 & 1.21 \\
\hline Gma.2505.1.S1_a_at & AB062754 & Ferritin & 1.74 & 1.42 & 2.88 & 4.26 & 3.11 & 2.21 \\
\hline GmaAffx.55267.1.S1_at & BE020105 & MATE efflux family protein & 1.19 & 1.24 & 1.76 & 7.84 & 1.22 & -1.35 \\
\hline GmaAffx.50677.1.S1_s_at & BI971744 & Proline-rich extensin-like family protein & 1.84 & 3.18 & 4.93 & 15.42 & 1.40 & 1.74 \\
\hline GmaAffx.91749.1.S1_s_at & CF807474 & Leucine-rich repeat transmembrane protein kinase & 1.48 & 2.08 & 20.03 & 5.99 & -1.94 & -4.54 \\
\hline Gma.11026.2.S1_s_at & BQ080041 & LeMir (miraculin homolog) trypsin and protease inhibitor & 1.20 & -1.31 & 11.84 & 17.61 & -1.76 & -2.12 \\
\hline Gma.5283.1.S1_at & BM091956 & Expansin family protein (EXPR3) & -1.06 & 4.86 & 6.79 & 75.66 & 11.67 & 91.38 \\
\hline GmaAffx.92774.1.S1_at & CF808124 & Polygalacturonase inhibiting protein 1 (PGIP1) & 1.46 & -1.09 & 3.36 & 5.72 & -1.06 & -1.06 \\
\hline Gma.4332.1.S1_at & AI442841 & Disease resistance-responsive family protein & 5.37 & 2.78 & 26.48 & 18.23 & 2.79 & 3.23 \\
\hline GmaAffx.60533.1.S1_at & BQ453624 & Laccase family protein & 2.08 & 1.87 & 3.95 & 10.32 & 1.49 & 2.88 \\
\hline GmaAffx.90538.1.A1_s_at & CF806263 & Pathogenesis-related protein 5 (PR-5) & 3.03 & 4.07 & 17.57 & 27.62 & 11.72 & 44.79 \\
\hline GmaAffx.88845.1.S1_at & CK605838 & Pathogenesis-related protein, similar to PR-1a protein & 1.55 & -1.08 & 16.29 & 20.26 & 8.11 & 10.48 \\
\hline Gma.4300.1.S1_at & S46989 & Chalcone synthase & 1.03 & -2.06 & 2.28 & 4.74 & 1.12 & 1.65 \\
\hline Gma.2499.1.S1_at & CD407677 & $\begin{array}{l}\text { Gibberellin-regulated family protein, similar to GAST1 } \\
\text { protein precursor }\end{array}$ & -1.19 & -1.58 & -1.68 & -1.85 & -1.34 & -1.56 \\
\hline Gma.7227.1.S1_at & BU551112 & Ubiquitin-conjugating enzyme 2 (UBC2) & -3.25 & -1.69 & -3.89 & -1.78 & -3.25 & -2.85 \\
\hline Gma.4837.1.S1_at & BI969343 & Nitrate transporter (NTP2) & -1.21 & -1.85 & -2.04 & -2.32 & -2.07 & -2.43 \\
\hline GmaAffx.32345.1.A1_s_at & BI970277 & Pectinesterase family protein & -2.29 & -5.2 & -1.83 & -1.15 & -3.05 & -4.34 \\
\hline Gma.7418.1.S1_at & BU547276 & Cytochrome P450 & -1.13 & -1.69 & -2.37 & -10.00 & -3.06 & 2.61 \\
\hline
\end{tabular}

${ }^{a}$ Fold changes for each gene at each timepoint in soybean cyst nematode (SCN)-infected roots were determined by comparison to corresponding mock samples. The qPCR results are compared with microarray results at each timepoint. Negative values indicate that the gene is suppressed in SCN-infected roots. Dark gray boxes indicate genes upregulated by at least 1.5-fold, Light gray boxes indicate genes downregulated by at least 1.5 -fold. The white boxes indicate genes with changes less than 1.5 -fold between two timepoints. 
Table 2. The 73 soybean cyst nematode gland-specific genes showing significant differential expression during development

\begin{tabular}{|c|c|c|c|c|c|c|c|c|}
\hline \multirow[b]{2}{*}{ Affymetrix probe ID } & \multirow{2}{*}{$\begin{array}{l}\text { GenBank } \\
\text { accession }\end{array}$} & \multirow{2}{*}{$\begin{array}{l}\text { Homolog } \\
\text { accessions }^{\text {a }}\end{array}$} & \multirow[b]{2}{*}{ Homolog description } & \multirow[b]{2}{*}{ E value } & \multirow[b]{2}{*}{$Q$ value } & \multicolumn{3}{|c|}{ Fold change $(\mathrm{dpi})^{\mathrm{b}}$} \\
\hline & & & & & & 2 to 5 & 5 to 10 & 2 to 10 \\
\hline \multicolumn{9}{|l|}{ Cluster 1} \\
\hline HgAffx.10157.1.S1_at & CB378344 & AAP30770 & DG pioneer protein 17G01 & $1 \mathrm{E}-27$ & 0.0002 & 4.46 & 17.15 & 76.56 \\
\hline HgAffx.13278.1.S1_at & CD748618 & AAR01198 & SvG cellulose binding protein $3 \mathrm{~B} 05$ & $5 \mathrm{E}-6$ & 0.0018 & 9.96 & 5.33 & 53.09 \\
\hline HgAffx.19469.1.SI_at & CB278984 & AAR01198 & SvG cellulose binding protein $3 \mathrm{~B} 05$ & $4 \mathrm{E}-9$ & 0.0004 & 5.50 & 13.38 & 73.63 \\
\hline HgAffx.22522.2.S1_x_at & CK351795 & AAL78214 & DG cell pioneer protein SB84 (Hgg-20) & $9 \mathrm{E}-8$ & 0.0041 & 4.56 & 7.60 & 34.65 \\
\hline HgAffx.2890.2.S1_at & CK351817 & AAL78214 & DG pioneer protein SB84 (Hgg-20) & $4 \mathrm{E}-16$ & 0.0009 & 5.71 & 7.05 & 40.27 \\
\hline \multicolumn{9}{|l|}{ Cluster 2} \\
\hline HgAffx.15851.1.S1_at & AF500034 & $\begin{array}{l}\text { AAP30773 } \\
\text { AAN32886 } \\
\text { AAP30754 }\end{array}$ & $\begin{array}{l}\text { DG pioneer protein } 24 \mathrm{Al} 2 \\
\text { DG pioneer protein } 2 \mathrm{D} 01 \\
\text { DG pioneer protein } 11 \mathrm{~A} 06\end{array}$ & $\begin{array}{l}5 \mathrm{E}-72 \\
6 \mathrm{E}-65 \\
3 \mathrm{E}-57\end{array}$ & 0.0019 & 25.65 & -1.16 & 22.18 \\
\hline HgAffx.15851.2.S1_x_at & AF500015 & $\begin{array}{l}\text { AAP30754 } \\
\text { AAN32886 } \\
\text { AAP30773 }\end{array}$ & $\begin{array}{l}\text { DG pioneer protein } 11 \mathrm{~A} 06 \\
\text { DG pioneer protein } 2 \mathrm{D} 01 \\
\text { DG pioneer protein } 24 \mathrm{~A} 12\end{array}$ & $\begin{array}{l}4 \mathrm{E}-75 \\
5 \mathrm{E}-72 \\
6 \mathrm{E}-49\end{array}$ & 0.0278 & 31.75 & -1.55 & 20.44 \\
\hline HgAffx.15851.3.SI_at & CD748340 & $\begin{array}{l}\text { AAP30754 } \\
\text { AAN32886 } \\
\text { AAP30773 }\end{array}$ & $\begin{array}{l}\text { DG pioneer protein } 11 \mathrm{~A} 06 \\
\text { DG pioneer protein } 2 \mathrm{D} 01 \\
\text { DG pioneer protein } 24 \mathrm{~A} 12\end{array}$ & $\begin{array}{l}2 \mathrm{E}-76 \\
2 \mathrm{E}-69 \\
3 \mathrm{E}-48\end{array}$ & 0.0011 & 6.07 & 1.24 & 7.54 \\
\hline HgAffx.19290.1.S1_x_at & CK348866 & AAL78214 & DG pioneer protein SB84 (Hgg-20) & $7 \mathrm{E}-10$ & 0.0098 & 3.27 & 1.45 & 4.73 \\
\hline HgAffx.23868.1.S1_at & CD748226 & AAR01198 & SvG cellulose binding protein $3 \mathrm{~B} 05$ & $2 \mathrm{E}-10$ & 0.0055 & 5.56 & 1.37 & 7.62 \\
\hline HgAffx.24001.1.S1_at & AF070225 & AAR85527 & $\begin{array}{l}\text { DG 14-3-3b protein [Meloidogyne } \\
\text { incognita] }\end{array}$ & $7 \mathrm{E}-13$ & 0.0298 & 2.03 & 1.22 & 2.48 \\
\hline HgAffx.24001.2.S1_at & CK351582 & AAR85527 & DG 14-3-3b protein $[M$. incognita $]$ & $2 \mathrm{E}-73$ & 0.0319 & 1.97 & 1.01 & 1.99 \\
\hline HgAffx.2849.1.S1_at & AF490248 & $\begin{array}{l}\text { AAO85456 } \\
\text { AAN32892 } \\
\text { AAP30755 }\end{array}$ & $\begin{array}{l}\text { DG pioneer protein } 18 \mathrm{H} 08 \\
\text { Secretory protein 4D06 } \\
\text { Putative gland protein 29D09 }\end{array}$ & $\begin{array}{l}3 \mathrm{E}-62 \\
3 \mathrm{E}-5 \\
9 \mathrm{E}-5\end{array}$ & 0.0136 & 4.21 & -1.46 & 2.89 \\
\hline HgAffx.4006.1.S1_at & CB825557 & AAN15806 & $\begin{array}{l}\text { SvG pioneer protein } 5 \mathrm{G} 05(\mathrm{SP} 26)[M \text {. } \\
\text { incognita }]\end{array}$ & $4 \mathrm{E}-40$ & 0.0494 & 1.83 & 1.10 & 2.00 \\
\hline \multicolumn{9}{|l|}{ Cluster 3} \\
\hline HgAffx.4037.1.S1_at & AF469058 & AAR01198 & SvG cellulose binding protein $3 \mathrm{~B} 05$ & $7 \mathrm{E}-61$ & 0.0011 & 23.42 & -2.43 & 9.64 \\
\hline HgAffx.4135.2.S1_at & AF500020 & $\begin{array}{l}\text { AAM50038 } \\
\text { AAO33476 } \\
\text { AAP30759 } \\
\text { AAP30760 } \\
\text { AAP30761 } \\
\end{array}$ & $\begin{array}{l}\text { DG pioneer protein 27D09 } \\
\text { DG pioneer protein } 6 \mathrm{E} 07 \\
\text { DG pioneer protein } 13 \mathrm{~A} 06 \\
\text { DG pioneer protein } 10 \mathrm{~A} 07 \\
\text { DG pioneer protein } 20 \mathrm{G} 04\end{array}$ & $\begin{array}{l}2 \mathrm{E}-39 \\
3 \mathrm{E}-40 \\
4 \mathrm{E}-40 \\
9 \mathrm{E}-27 \\
2 \mathrm{E}-25 \\
\end{array}$ & 0.0072 & 9.69 & -4.73 & 2.05 \\
\hline \multicolumn{9}{|l|}{ Cluster 4} \\
\hline HgAffx.19033.1.S1_x_at & AF500028 & $\begin{array}{l}\text { AAO85453 } \\
\text { AAP30767 }\end{array}$ & $\begin{array}{l}\text { SvG pioneer protein } 16 \mathrm{~A} 01 \\
\text { DG pioneer protein } 21 \mathrm{E} 12\end{array}$ & $\begin{array}{l}4 \mathrm{E}-5 \\
3 \mathrm{E}-4\end{array}$ & 0.0303 & -2.89 & 9.05 & 3.14 \\
\hline HgAffx.2243.1.S1_at & CK351480 & AAL78214 & DG pioneer protein SB84 (Hgg-20) & $1 \mathrm{E}-17$ & 0.0122 & -4.30 & 44.06 & 10.24 \\
\hline HgAffx.22522.2.Al_x_at & CK351795 & AAL78214 & DG pioneer protein SB84 (Hgg-20) & $9 \mathrm{E}-8$ & 0.0396 & -6.18 & 30.88 & 5.00 \\
\hline HgAffx.10011.1.S1_at & CB378490 & AAP30770 & DG pioneer protein $17 \mathrm{G} 01$ & $1 \mathrm{E}-13$ & 0.0234 & -1.45 & 6.62 & 4.58 \\
\hline HgAffx.11249.1.S1_at & CB935201 & AAP30770 & DG pioneer protein $17 \mathrm{G} 01$ & $4 \mathrm{E}-9$ & 0.0067 & -1.48 & 10.61 & 7.19 \\
\hline HgAffx.16883.1.S1_at & CB279474 & AAL78214 & DG pioneer protein SB84 Hgg-20 & $9 \mathrm{E}-13$ & 0.0034 & 1.10 & 28.53 & 31.35 \\
\hline HgAffx.19033.1.S1_s_at & AF500028 & $\begin{array}{l}\text { AAO85453 } \\
\text { AAP30767 }\end{array}$ & $\begin{array}{l}\text { SvG pioneer protein } 16 \mathrm{~A} 01 \\
\text { DG pioneer protein } 21 \mathrm{E} 12\end{array}$ & $\begin{array}{l}4 \mathrm{E}-5 \\
3 \mathrm{E}-4\end{array}$ & 0.0002 & -1.67 & 597.88 & 358.60 \\
\hline HgAffx.19033.2.S1_at & AF500027 & $\begin{array}{l}\text { AAP30766 } \\
\text { AAO85453 } \\
\text { AAP30767 }\end{array}$ & $\begin{array}{l}\text { DG pioneer protein } 30 \mathrm{D} 08 \\
\text { DG pioneer protein } 16 \mathrm{~A} 01 \\
\text { DG pioneer protein } 21 \mathrm{E} 12\end{array}$ & $\begin{array}{l}2 \mathrm{E}-11 \\
6 \mathrm{E}-7 \\
2 \mathrm{E}-6\end{array}$ & 0.0130 & -1.16 & 91.77 & 79.42 \\
\hline HgAffx.19033.4.S1_s_at & CK350593 & $\begin{array}{l}\text { AAP30766 } \\
\text { AAP30767 } \\
\text { AAO85453 }\end{array}$ & $\begin{array}{l}\text { DG pioneer protein } 30 \mathrm{D} 08 \\
\text { DG pioneer protein } 21 \mathrm{E} 12 \\
\text { DG pioneer protein } 16 \mathrm{~A} 01\end{array}$ & $\begin{array}{l}4 \mathrm{E}-5 \\
4 \mathrm{E}-5 \\
4 \mathrm{E}-5\end{array}$ & 0.0219 & -1.09 & 16.11 & 14.83 \\
\hline HgAffx.1945.1.S1_at & CK351851 & AAL78214 & DG pioneer protein SB84 (Hgg-20) & $6 \mathrm{E}-4$ & 0.0136 & -1.13 & 12.26 & 10.88 \\
\hline HgAffx.22522.2.S1_at & CK351795 & AAL78214 & DG pioneer protein SB84 (Hgg-20) & $9 \mathrm{E}-8$ & 0.0194 & 1.35 & 10.05 & 13.60 \\
\hline HgAffx.2890.1.S1_at & CK 350408 & AAL78214 & DG pioneer protein SB84 (Hgg-20) & $8 \mathrm{E}-81$ & 0.0276 & 1.36 & 14.89 & 20.28 \\
\hline HgAffx.2890.2.S1_s_at & CK351817 & AAL78214 & DG pioneer protein SB84 (Hgg-20) & $6 \mathrm{E}-36$ & 0.0024 & 1.06 & 53.53 & 56.88 \\
\hline HgAffx.3143.1.S1_at & CK351245 & AAL78214 & DG pioneer protein SB84 (Hgg-20) & $7 \mathrm{E}-6$ & 0.0379 & 1.54 & 3.43 & 5.28 \\
\hline HgAffx.5473.1.S1_at & CD747951 & AAR85527 & DG $14-3-3 b$ protein $[M$. incognita $]$ & $\mid \mathrm{E}-41$ & 0.0028 & -1.25 & 5.24 & 4.20 \\
\hline HgAffx.6623.1.S1_at & CB825588 & AAR85527 & DG $14-3-3 b$ protein $[M$. incognita $]$ & $6 \mathrm{E}-39$ & 0.0126 & -1.86 & 11.47 & 6.15 \\
\hline HgAffx.6660.1.S1_at & CB825551 & AAP30770 & DG pioneer protein $17 \mathrm{G} 01$ & $2 \mathrm{E}-24$ & 0.0012 & 1.53 & 56.62 & 86.54 \\
\hline HgAffx.9769.1.S1_at & CB378732 & AAP30770 & DG pioneer protein $17 \mathrm{G} 01$ & $2 \mathrm{E}-18$ & 0.0069 & 1.12 & 54.29 & 60.94 \\
\hline HgAffx.7996.1.S1_at & CB824215 & AAP30770 & DG pioneer protein $17 \mathrm{G} 01$ & IE-18 & 0.0040 & 2.15 & 27.77 & 59.59 \\
\hline & & & & & & & (Contin & on next \\
\hline
\end{tabular}

${ }^{a}$ BLASTX hits. All homologs are from Heterodera glycines unless indicated otherwise. Protein accession numbers and E-values are given for all the significant BLASTX matches to gland cell-specific genes. DG = dorsal gland; SvG = subventral gland.

${ }^{\mathrm{b}}$ Dark gray boxes indicate genes upregulated by at least 1.5 -fold. Light gray boxes indicate genes downregulated by at least 1.5 -fold. The white boxes indicate genes with changes less than 1.5 -fold between two timepoints. 
Table 2. (Continued from preceding page)

\begin{tabular}{|c|c|c|c|c|c|c|c|c|}
\hline \multirow[b]{2}{*}{ Affymetrix probe ID } & \multirow{2}{*}{$\begin{array}{l}\text { GenBank } \\
\text { accession }\end{array}$} & \multirow{2}{*}{$\begin{array}{c}\text { Homolog } \\
\text { accessions }^{\mathrm{a}}\end{array}$} & \multirow[b]{2}{*}{ Homolog description } & \multirow[b]{2}{*}{ E value } & \multirow[b]{2}{*}{$Q$ value } & \multicolumn{3}{|c|}{ Fold change $(\mathrm{dpi})^{\mathrm{b}}$} \\
\hline & & & & & & 2 to 5 & 5 to 10 & 2 to 10 \\
\hline \multicolumn{9}{|l|}{ Cluster 5} \\
\hline HgAffx.17301.1.S1_at & CB935153 & AAL 78214 & DG cell pioneer protein SB84 (Hgg-20) & $1 \mathrm{E}-10$ & 0.0372 & 2.63 & 2.30 & 6.04 \\
\hline HgAffx.19033.4.Al_at & CK350593 & $\begin{array}{l}\text { AAP30766 } \\
\text { AAP30767 } \\
\text { AAO85453 }\end{array}$ & $\begin{array}{l}\text { DG pioneer protein } 30 \mathrm{D} 08 \\
\text { DG pioneer protein } 21 \mathrm{E} 12 \\
\text { DG pioneer protein } 16 \mathrm{~A} 01\end{array}$ & $\begin{array}{l}4 \mathrm{E}-5 \\
4 \mathrm{E}-5 \\
4 \mathrm{E}-5\end{array}$ & 0.0418 & -1.45 & -1.72 & -2.49 \\
\hline HgAffx.22856.1.S1_at & AF006052 & AAC48327 & SvG beta-1,4-endoglucanase-1 & $2 \mathrm{E}-29$ & 0.0396 & -1.63 & -1.73 & -2.83 \\
\hline HgAffx.4139.2.A1_x_at & CK348925 & AAP30775 & DG pioneer protein $32 \mathrm{E} 03$ & $1 \mathrm{E}-10$ & 0.0425 & -1.96 & -2.78 & -5.46 \\
\hline HgAffx.2032.1.S1_at & CB378359 & AAL78214 & DG pioneer protein SB84 (Hgg-20) & $2 \mathrm{E}-14$ & 0.0350 & 2.54 & 3.30 & 8.37 \\
\hline HgAffx.3706.1.S1_at & CB374919 & AAL 40720 & SvG/DG calreticulin $[M$. incognita $]$ & $5 \mathrm{E}-40$ & 0.0487 & 1.57 & 1.68 & 2.64 \\
\hline \multicolumn{9}{|l|}{ Cluster 6} \\
\hline HgAffx.16985.1.S1_at & CB279371 & $\begin{array}{l}\text { AAP30757 } \\
\text { AAO33477 } \\
\text { AAP30758 }\end{array}$ & $\begin{array}{l}\text { DG pioneer protein } 30 \mathrm{G} 12 \\
\text { DG pioneer protein } 4 \mathrm{G} 05 \\
\text { Putative gland protein } 25 \mathrm{~A} 01\end{array}$ & $\begin{array}{l}2 \mathrm{E}-11 \\
2 \mathrm{E}-11 \\
2 \mathrm{E}-11\end{array}$ & 0.0169 & -1.91 & -2.87 & -5.49 \\
\hline HgAffx.20336.1.S1_at & AF044210 & AAC15708 & SvG beta-1,4-endoglucanase-2 & $4 \mathrm{E}-26$ & 0.0106 & -2.04 & -4.78 & -9.75 \\
\hline HgAffx.21086.1.S1_at & AF490250 & AAO 85458 & DG pioneer protein $19 \mathrm{C} 07$ & $4 \mathrm{E}-43$ & 0.0108 & -1.78 & -2.66 & -4.74 \\
\hline HgAffx.22005.4.S1_x_at & AF344863 & $\begin{array}{l}\text { AAL78212 } \\
\text { AAP30776 }\end{array}$ & $\begin{array}{l}\text { DG pioneer protein SB89 }(\mathrm{Hgg}-25) \\
\text { DG pioneer protein } 34 \mathrm{~B} 08\end{array}$ & $\begin{array}{l}1 \mathrm{E}-47 \\
4 \mathrm{E}-27\end{array}$ & 0.0123 & -1.77 & -2.44 & -4.32 \\
\hline HgAffx.3020.1.S1_at & AF468679 & AAN14978 & SvG chitinase 3D11 & $4 \mathrm{E}-63$ & 0.0026 & -1.28 & -2.45 & -3.13 \\
\hline HgAffx.4275.4.S1_s_at & AF345800 & $\begin{array}{l}\text { AAL78228 } \\
\text { AAP30764 }\end{array}$ & $\begin{array}{l}\text { SvG pioneer protein GSB3 }(\mathrm{Hgg}-24) \\
\text { DG pioneer protein } 28 \mathrm{~B} 03\end{array}$ & $\begin{array}{l}3 \mathrm{E}-130 \\
2 \mathrm{E}-94\end{array}$ & 0.0039 & -1.53 & -3.51 & -5.37 \\
\hline HgAffx.3578.1.S1_at & AF469059 & AAN32888 & DG annexin $4 \mathrm{~F} 01$ & $3 \mathrm{E}-60$ & 0.0325 & 1.43 & -2.10 & -1.47 \\
\hline HgAffx.14339.1.S1_at & AF490246 & $\begin{array}{l}\text { AAP30774 } \\
\text { AAO85454 } \\
\text { AAP30768 }\end{array}$ & $\begin{array}{l}\text { DG pioneer protein } 30 \mathrm{E} 03 \\
\text { DG pioneer protein } 16 \mathrm{~B} 09 \\
\text { DG pioneer protein } 22 \mathrm{C} 12\end{array}$ & $\begin{array}{l}1 \mathrm{E}-88 \\
1 \mathrm{E}-88 \\
4 \mathrm{E}-84\end{array}$ & 0.0243 & 1.04 & -3.05 & -2.93 \\
\hline HgAffx.20813.1.S1_at & AF502393 & AAP30836 & DG pioneer protein $30 \mathrm{C} 02$ & $2 \mathrm{E}-77$ & 0.0242 & 1.33 & -3.59 & -2.71 \\
\hline HgAffx.22770.1.S1_at & AF469060 & AAN32889 & DG ubiquitin extension protein $4 \mathrm{G} 06$ & $7 \mathrm{E}-44$ & 0.0327 & -1.15 & -2.13 & -2.46 \\
\hline HgAffx.22811.1.S1_at & AF343567 & AAL73347 & $\begin{array}{l}\text { SvG venom-allergen protein (Vap-1) } \\
\text { GSB9 (SP13) }\end{array}$ & $1 \mathrm{E}-65$ & 0.0256 & -1.27 & -2.89 & -3.67 \\
\hline HgAffx.24067.1.S1_at & AF520565 & AAO63754 & DG chorismate mutase 45D07 & $1 \mathrm{E}-80$ & 0.0041 & -1.30 & -3.97 & -5.14 \\
\hline HgAffx.3111.1.S1_at & AF500026 & AAP30765 & SvG pioneer protein $15 \mathrm{~A} 10$ & $5 \mathrm{E}-73$ & 0.0029 & 1.05 & -4.18 & -3.97 \\
\hline HgAffx.3699.1.S1_s_at & AF473827 & $\begin{array}{l}\text { AAO33474 } \\
\text { AAG21331 }\end{array}$ & $\begin{array}{l}\text { DG CLE protein } 4 \mathrm{G} 12 \\
\text { DG CLE protein SYV46 (SP1) }\end{array}$ & $\begin{array}{l}1 \mathrm{E}-58 \\
1 \mathrm{E}-56\end{array}$ & 0.0115 & -1.27 & -2.22 & -2.82 \\
\hline HgAffx.4135.1.S1_at & AF500021 & $\begin{array}{l}\text { AAM50038 } \\
\text { AAP30761 } \\
\text { AAP30760 }\end{array}$ & $\begin{array}{l}\text { DG pioneer protein 27D09 } \\
\text { DG pioneer protein } 20 \mathrm{G} 04 \\
\text { DG pioneer protein } \mathrm{G} 10 \mathrm{~A} 07\end{array}$ & $\begin{array}{l}3 \mathrm{E}-31 \\
2 \mathrm{E}-74 \\
2 \mathrm{E}-73\end{array}$ & 0.0470 & 1.40 & -3.68 & -2.62 \\
\hline HgAffx.4135.3.Al_at & CK349109 & AAM50038 & DG pioneer protein 27D09 & $11 \mathrm{E}-3$ & 0.0475 & 1.28 & -9.32 & -7.30 \\
\hline HgAffx.4139.1.S1_at & AF500036 & AAP30775 & DG pioneer protein $32 \mathrm{E} 03$ & $4 \mathrm{E}-32$ & 0.0124 & 1.10 & -2.52 & -2.28 \\
\hline HgAffx.4275.1.S1_at & AF500025 & $\begin{array}{l}\text { AAP30764 } \\
\text { AAL78219 } \\
\text { AAL78228 }\end{array}$ & $\begin{array}{l}\text { DG pioneer protein 28B03 } \\
\text { DG pioneer protein SB26 (Hgg-26) } \\
\text { SvG pioneer protein GSB3 (Hgg-24) }\end{array}$ & $\begin{array}{l}8 \mathrm{E}-123 \\
2 \mathrm{E}-117 \\
6 \mathrm{E}-66\end{array}$ & 0.0161 & -1.55 & -2.74 & -4.25 \\
\hline HgAffx.4275.2.S1_at & AF500024 & AAP30763 & DG SKP-1 like protein $8 \mathrm{H} 07$ & $3 \mathrm{E}-166$ & 0.0212 & -1.47 & -2.55 & -3.75 \\
\hline HgAffx.4275.4.A1_at & AF345800 & $\begin{array}{l}\text { AAL78228 } \\
\text { AAP30764 } \\
\end{array}$ & $\begin{array}{l}\text { SvG pioneer protein GSB3 }(\mathrm{Hgg}-24) \\
\text { DG pioneer protein } 28 \mathrm{~B} 03\end{array}$ & $\begin{array}{l}3 \mathrm{E}-130 \\
2 \mathrm{E}-94 \\
\end{array}$ & 0.0236 & -1.63 & -4.65 & -7.58 \\
\hline \multicolumn{9}{|l|}{ Cluster 8} \\
\hline HgAffx.22005.5.S1_s_at & BF013581 & $\begin{array}{l}\text { AAL78212 } \\
\text { AAP30776 }\end{array}$ & $\begin{array}{l}\text { DG pioneer protein SB89 (Hgg-25) } \\
\text { DG pioneer protein } 34 \mathrm{~B} 08\end{array}$ & $\begin{array}{l}2 \mathrm{E}-25 \\
9 \mathrm{E}-16\end{array}$ & 0.0157 & -3.01 & -1.86 & -5.60 \\
\hline HgAffx.2130.1.S1_at & AY101191 & AAM50039 & SvG cellulase 26D05 & 0 & 0.0222 & -4.22 & -1.91 & -8.05 \\
\hline HgAffx.22005.2.S1_at & AF500037 & AAP30776 & DG pioneer protein $34 \mathrm{~B} 08$ & $1 \mathrm{E}-46$ & 0.0278 & -3.31 & -1.92 & -6.36 \\
\hline HgAffx.22045.1.S1_at & BG310823 & AAM21970 & $\begin{array}{l}\text { SvG pectate lyase } 2 \text { [Globodera } \\
\text { rostochiensis] }\end{array}$ & $3 \mathrm{E}-23$ & 0.0409 & -5.20 & -2.28 & -11.88 \\
\hline HgAffx.23342.1.S1_at & $\mathrm{BF} 014507$ & CAC83611 & $\begin{array}{l}\text { SvG expansin EXPB1 [Globodera } \\
\text { rostochiensis] }\end{array}$ & $7 \mathrm{E}-39$ & 0.0230 & -6.76 & -2.33 & -15.72 \\
\hline HgAffx.99.1.A1_at & AF273735 & AAG21337 & DG protein SYV42 (SP8) & $3 \mathrm{E}-55$ & 0.0067 & -2.46 & -1.24 & -3.06 \\
\hline \multicolumn{9}{|l|}{ Cluster 9} \\
\hline HgAffx.20336.2.S1_s_at & AY043224 & AAP88024 & SvG beta-1,4-endoglucanase- 4 & $2 \mathrm{E}-79$ & 0.0022 & -2.40 & -11.78 & -28.29 \\
\hline HgAffx.22005.1.S1_at & AF502392 & $\begin{array}{l}\text { AAP30835 } \\
\text { AAL78211 } \\
\text { AAP30776 }\end{array}$ & $\begin{array}{l}\text { DG proline-rich glycoprotein 33E05 } \\
\text { proline-rich glycoprotein GSB24 (Hgg-15) } \\
\text { DG pioneer protein } 34 \text { B } 08\end{array}$ & $\begin{array}{l}5 \mathrm{E}-125 \\
9 \mathrm{E}-54 \\
4 \mathrm{E}-34\end{array}$ & 0.0006 & -3.55 & -2.57 & -9.12 \\
\hline HgAffx.22014.1.S1_at & AF469061 & AAN32890 & DG pioneer protein 4D09 & $6 \mathrm{E}-46$ & 0.0011 & -2.45 & -3.95 & -9.68 \\
\hline HgAffx.3764.1.S1_at & AF473830 & $\begin{array}{l}\text { AAO33477 } \\
\text { AAP30757 }\end{array}$ & $\begin{array}{l}\text { DG pioneer protein } 4 \mathrm{G} 05 \\
\text { DG pioneer protein } 30 \mathrm{G} 12\end{array}$ & $\begin{array}{l}5 \mathrm{E}-100 \\
3 \mathrm{E}-91\end{array}$ & 0.0087 & -2.34 & -2.16 & -5.05 \\
\hline HgAffx.3764.3.S1_s_at & CK350050 & $\begin{array}{l}\text { AAO33477 } \\
\text { AAP30757 }\end{array}$ & $\begin{array}{l}\text { DG pioneer protein } 4 \mathrm{G} 05 \\
\text { DG pioneer protein } 30 \mathrm{G} 12\end{array}$ & $\begin{array}{l}2 \mathrm{E}-26 \\
3 \mathrm{E}-39\end{array}$ & 0.0042 & -2.52 & -3.39 & -8.54 \\
\hline HgAffx.5088.1.S1_at & AF473826 & $\begin{array}{l}\text { AAP30756 } \\
\mathrm{AAO} 33473\end{array}$ & $\begin{array}{l}\text { SvG pioneer protein } 10 \mathrm{C} 02 \\
\mathrm{SvG} \text { nioneer nrotein } 4 \mathrm{~F} 02\end{array}$ & $\begin{array}{l}8 \mathrm{E}-37 \\
8 \mathrm{E}-37 \\
\end{array}$ & 0.0018 & -2.21 & -1.85 & -4.08 \\
\hline
\end{tabular}


upregulated from 2 to $5 \mathrm{dpi}$ and then significantly downregulated from 5 to $10 \mathrm{dpi}$. Transcripts in cluster 7 exhibit the opposite pattern. Cluster 5 includes transcripts that exhibited significantly nonconstant expression across the three timepoints but with no significant changes in expression between adjacent time points. Thus, the transcripts in this cluster tend to increase or decrease more gradually over the time course than transcripts in either cluster 1 or 9 .

Five differentially expressed genes were selected randomly for validation by qPCR. The transcript details and the primer sequences are given in Supplemental Table 2. For comparison, RNA from freshly hatched preparasitic J2 also was included in qPCR studies. A SCN GAPDH (CA939315) gene was used as an internal control for the relative quantification of gene expression using the $\Delta \Delta \mathrm{C}_{\mathrm{T}}$ method (described below). This SCN $G A P D H$ was used as one of the internal controls on the chip. Our microarray analysis showed that this gene was upregulated from 2 to $5 \mathrm{dpi}$ and stayed constant between 5 and 10 dpi. However, our qPCR analysis using a known concentration of total RNA from different preparasitic and parasitic stages of SCN indicated that the expression of SCN GAPDH was stable across all of the nematode developmental stages tested (data not shown). This discrepancy likely is due to cross hybridization of other $G A P D H$ family transcripts in microarray analysis. The qPCR results were consistent with the microarray results for all five genes selected (Fig. 3). The transcript level for a gene encoding a putative cuticular collagen (HgAffx.13886.1.S1_at) was very low in preparasitic $\mathrm{J} 2 \mathrm{~s}$ and went up with the development of parasitic stages, reaching a peak at 5 dpi. A similar expression pattern was observed for a gene encoding putative esophageal gland cell protein Hgg-20 (HgAffx.2890.2.S1_at); however, the transcript level peaked at $10 \mathrm{dpi}$. Transcript levels for genes encoding chorismate mutase (HgAffx.24067.1.S1_at) and a putative FMRFamide-related peptide (HgAffx.23446.1. $\mathrm{S} 1 \_$at) precursor were high in preparasitic J2s but consistently went down with the development of the nematode. In contrast,

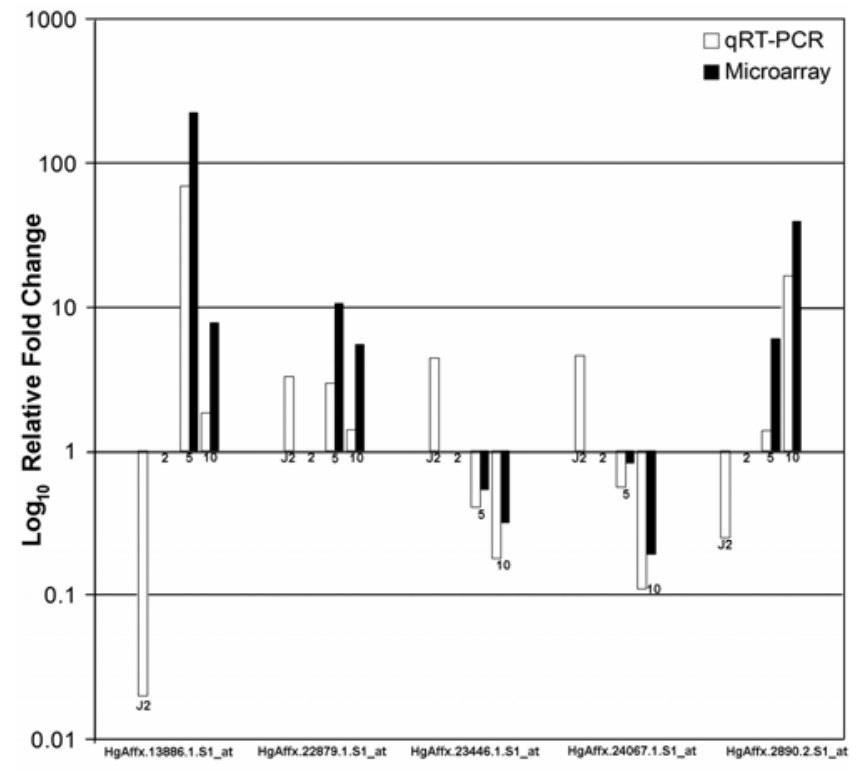

Affymetrix Probe ID

Fig. 3. Quantitative real-time reverse-transcription polymerase chain reaction (qPCR) confirmation of microarray analysis for soybean cyst nematode gene expression. Total RNA was isolated from roots harvested at 2, 5, and 10 days postinoculation (dpi). Total RNA from preparasitic secondstage juveniles (J2) also was used for comparison. First-strand cDNA was synthesized and subjected to qPCR in triplicate. Values on the $\mathrm{Y}$ axis indicate $\log _{10}$-fold change relative to the 2 dpi timepoint. The qPCR results are compared with microarray results at 2,5 , and $10 \mathrm{dpi}$. expression of a gene encoding a putative secreted glutathione peroxidase (HgAffx.22879.1.S1_at) was high in preparasitic J2s, downregulated at $2 \mathrm{dpi}$, and then went up during development of the parasitic nematode, to reach a peak at $5 \mathrm{dpi}$.

\section{DISCUSSION}

The genome-wide gene expression analysis conducted in this study represents the first successful example of using a single microarray to simultaneously determine gene expression profiles in a nematode and plant over a time course of the infection process. The identification of genes regulated in both the host and pathogen provides novel insights into the complex nature of the SCN-soybean pathosystem, which includes a transit from migratory to sedentary life stages and the establishment of a unique feeding structure in host plant roots. Using Affymetrix GeneChip soybean genome arrays, we have identified the most comprehensive list of transcripts regulated in both soybean and SCN during parasitism to date. Our results provide insight into the complex changes in plant gene expression occurring globally in response to $\mathrm{SCN}$ parasitism, and have identified coordinated expression of nematode genes potentially involved in parasitism and specific stages of development.

Simultaneous analysis of changes in mesenger (m)RNA levels of 35,611 soybean transcripts and 7,431 SCN transcripts identified 429 soybean genes with significant changes in expression between SCN-infected and mock-inoculated roots and 1,850 SCN genes with significant changes in expression during parasitism when controlling FDR at approximately 5\% and using a 1.5 -fold cutoff. A subset of the 429 differentially expressed soybean genes was validated by qPCR. Many of the soybean genes differentially regulated by SCN infection were involved in metabolism, cell wall modification, stress or defense responses, phytohormone response, and cellular signaling. It is difficult to make direct comparisons with earlier microarray studies of cyst nematode-plant interactions due to differences in pathosystems, microarray platforms, time point selection, tissue sampling methods, and analysis; however, we point out a few similarities with the studies here. Khan and associates (2004) identified the upregulation of 99 genes and downregulation of one gene at 2 dpi in nematode-infected roots of a susceptible soybean line using a cDNA array with approximately 1,300 probes. Our data was consistent with this study for genes encoding peroxidase and extensin. Similarly, our data is consistent with an earlier microarray study of the Arabidopsisbeet cyst nematode interaction at 3 dpi (Puthoff et al. 2003) for genes encoding PR1, heat shock proteins, carboxypeptidases, glutathione- $S$-transferases (GSTs), expansins, extensins, pectinesterases, hexose transporter, nodulin, an ERF, and a WRKY family transcription factor. Our data extend their observations by measuring thousands more soybean and nematode genes simultaneously.

Changes in the expression of cell-wall-modifying proteins (CWMPs) have been shown to be important for cyst nematode-plant interactions. During penetration and intracellular migration through root tissues, cyst nematode juveniles secrete a mixture of CWMPs, including endoglucanases, pectate lyases, and expansins, to loosen cell walls (De Boer et al. 1999, 2002a; Goellner et al. 2000, 2001; Qin et al. 2004). Once a cyst nematode juvenile begins to feed from the initial syncytial cell, expression of nematode CWMPs declines (De Boer et al. 1999; Goellner et al. 2000, 2001) and the coordinated expression of plant CWMPs (Goellner et al. 2001) is activated for syncytium formation. This trend was reflected in our microarray study. We identified the differential regulation of nematode CWMPs, including $\beta$-1,4-endoglucanases, pec- 
tate lyase, and expansin, as well as soybean expansins, extensins, pectinesterases, and a xyloglucan endotransglycosylase during nematode parasitism of soybean. All of the nematode CWMPs, with the exception of a homolog of $\beta$-1,4-endoglucanase, fell into clusters 6,8 , and 9 , which represent genes with a significantly decreasing pattern of expression over the course of infection, and this reflects their importance during the early stages of the nematode life cycle. In contrast, all CWMPs of plant origin, including extensins and expansins, exhibited a significantly increasing pattern of expression over time, with the exception of a xyloglucan endotransglycosylase homolog and two homologs of pectinesterase family proteins, which showed an opposite trend. Differential regulation of major CWMPs, including expansins, pectate lyases, xyloglucan endotransglycosylases, and polygalacturonases, also was observed in root-knot nematode-induced galls in Arabidopsis (Jammes et al. 2005).

Our study also suggests general activation of plant defense mechanisms in response to SCN infection. Defense-related genes, including PR proteins, stress-related transcription factors (e.g., ERFs), general stress-responsive genes (e.g., glutathione$S$-transferases and osmotins), disease resistance responsive proteins, PGIPs, and homologs of a hypersensitive response (HR)-related gene, harpin-induced gene 1 (Hinl), were upregulated in nematode-infected roots. Upregulation of the hin family of genes has been observed in plants in response to bacterial (Gopalan et al. 1996) and fungal (Kim et al. 2000; Tian et al. 2003) pathogens as well as in response to symbionts (Le Quere et al. 2005). The general upregulation of plant defense responses in our study is opposite to the general suppression of plant defense mechanisms reported in the Arabidopsis-root knot nematode interaction (Jammes et al. 2005). This is not surprising considering that, unlike cyst nematodes which migrate intracellularly causing migration damage, root-knot nematodes migrate intercellularly and do not elicit a plant defense response along the path of migration. The earliest time point profiled by Jammes and associates (2005) was $7 \mathrm{dpi}$, at which time nematodes are sedentary within the developing gall. It is very possible that, in our study, suppression of general plant defense mechanisms at the nematode feeding site was masked by upregulation of defense-related genes due to wound-related responses in surrounding tissue caused by intracellular migration and, at later stages, as adult female nematodes expanded and eventually protruded from the root. This observation is consistent with our cluster analysis of differentially expressed plant genes, where all genes with an annotated function in the cluster combination mock cluster 5-infected cluster 2 appeared to be involved in plant defense responses. Genes in this cluster combination were significantly upregulated from 2 to 5 dpi but did not show any significant change between 5 and 10 dpi in nematode-infected roots. Expression of these genes did not change significantly between adjacent time points in the uninfected roots, consistent with the global activation of plant defense genes in response to nematode infection.

Genes encoding enzymes involved in secondary metabolism, specifically the phenylpropanoid and flavonoid pathways, including $4 c l$ (4-coumarate-CoA ligase), Chs (chalcone synthase), Chi (chalcone isomerase), $D f r$ (dihydroflavonol reductase), Ifr (isoflavone reductase), and a lignin biosynthetic pathway gene encoding quercetin 3-O-methyltransferase (OMT), were upregulated in nematode-infected roots. Upregulation of genes in the multibranched phenylpropanoid pathway leading to the accumulation of flavonoid or isoflavonoid phytoalexins, deposition of lignin, or cell wall bound phenolics is also a characteristic of the HR and systemic acquired resistance (SAR) in plant-pathogen interactions (Keen 1992). A study by Edens and associates (1995) showed that the transcript abundance for genes encoding phenylalanine ammonia lyase (PAL) and 4coumaryl CoA ligase as well as the activity of these two enzymes increased in resistant but not susceptible soybean cultivars after SCN or root-knot nematode infection. Synthesis of enzymes later in the phenylpropanoid pathway leading to glyceollin production also increased in both resistant and susceptible soybean in response to nematode infection, with a greater increase observed in resistant cultivars. The role of these secondary metabolites in compatible plant-nematode interactions is not clear. However, alterations in auxin distribution have been observed in nematode-infected roots and it has been suggested that increased flavonoid levels in response to infection may be involved in the local accumulation of auxin at feeding sites by blocking auxin efflux carriers (Hutangura et al. 1999). Consistent with this observation we see upregulation of an auxin-induced soybean gene, PIN2, encoding an auxin transport protein, indicating possible accumulation of auxin in SCN-infected soybean roots. In tomato and Arabidopsis, auxin has been shown to mediate the induction and morphogenesis of cyst nematode syncytia, and a strong reduction in cyst nematode development was observed on Arabidopsis pin2 mutants (Goverse et al. 2000).

The 1,850 SCN genes displaying statistically significant changes in expression over the time course of infection were grouped into nine clusters based on similarity of significant expression changes across adjacent time points. Significantly, the clustering analysis revealed coordinated expression of several classes of nematode genes. Our interest in SCN parasitism genes led us to further analyze the temporal expression patterns of nematode genes represented on the Affymetrix GeneChip soybean genome array that previously were confirmed to contain a signal peptide and shown to be expressed in the nematode esophageal gland cells (Table 2). Interestingly, the majority of previously identified SCN parasitism genes (both candidate genes and those shown to be secreted with known function) (Gao et al. 2001, 2003; Lambert et al. 1999; Smant et al. 1998; Wang et al. 2001) fell into clusters 6, 8, and 9, which include transcripts whose expression levels decrease significantly over the course of infection. In cluster 9, ENG-4 (endoglucanase; Gao et al. 2004) and the pioneer genes GSB24 (Hgg-15; Gao et al., 2001), 33E05, 34B08, 4D09, and 30 G12/4G05 and 10C02/4E02 gene families (Gao et al. 2003) displayed a high level of mRNA abundance at 2 dpi that declined at $5 \mathrm{dpi}$ and again at $10 \mathrm{dpi}$, suggesting that these genes may play a role in the early stages of the parasitic interaction. Similarly, in cluster 8, cellulase (26D05), PEL (pectate lyase; De Boer et al. 2002a), a gene with similarity to Globodera rostochiensis expansin (Qin et al. 2004), and dorsal gland pioneer genes 34B08 (Gao et al. 2003), SYV42 (Wang et al. 2001), and SB89 (Hgg-25, Gao et al. 2001) were present. In cluster 8, expression levels dropped significantly from 2 to $5 \mathrm{dpi}$ and then showed no significant change from 5 to 10 dpi. $E N G-2$ (Gao et al. 2004); CHT (chitinase; Gao et al. 2002); a putative annexin (ANN; 4FO1); $C M$ (chorismate mutase; 45D07; Gao et al. 2003); CLAVATA3/ESR-like (CLE; 4G12 [Gao et al. 2003] and $S Y V 46 / 2 B 10$ [Gao et al. 2003; Wang et al. 2001]); VAP (venom-allergen protein; Gao et al. 2001); ubiquitin extension protein (4G06); SKPl-like (8H07, 28B03, 29F07, GSB3, $S B 26)$; pioneers genes 19C07, 30CO2, 15A10, and 32E03; and 16B09/30E03/22C12, 27D09/20G04/10A07, and 30G12/4G05/ $25 A 01$ gene families fell into cluster 6 (Gao et al. 2003). SB89 and 34B08 also fell into cluster 6. Transcripts in cluster 6 showed no significant change in expression between 2 and 5 dpi but then dropped significantly from 5 to $10 \mathrm{dpi}$. The temporal expression profile suggests that these genes also play an important role in the earlier stages of the plant-nematode 
interaction, but remain expressed at higher levels longer than those present in clusters 8 and 9 .

A small subset of candidate SCN parasitism genes fell into clusters other than 6,8 , and 9. Two SCN parasitism genes fell into cluster 3 , including a cellulose-binding protein $(C B P)$ and the pioneer protein gene family 6E07/27D09 (Gao et al. 2003). Genes in cluster 3 showed an increase in expression level from 2 to $5 \mathrm{dpi}$, but declined from 5 to $10 \mathrm{dpi}$. SCN parasitism genes falling into clusters 1 and 2 included SB84 (Hgg-20; Gao et al. 2001), CBP, 17G01, 2D01/11A06/24A12, and $4 D 06 / 18 H 08 / 29 D 09$ pioneer protein gene families (Gao et al. 2003); a gene similar to Meloidogyne incognita subventral gland pioneer protein gene $5 G 05$ (Huang et al. 2003); and an M. incognita dorsal gland 14-3-3b gene (Jaubert et al. 2004). Genes in clusters 1 and 2 showed an increase in expression from 2 to $5 \mathrm{dpi}$, but either increased from 5 to $10 \mathrm{dpi}$ (cluster 1) or showed no significant change from 5 to $10 \mathrm{dpi}$ (cluster 2). Nematode genes falling into clusters 1,2 , and 3 may play a more predominant role during later stages of the plant-nematode interaction. SB84 (Hgg-20), 17G01, and a gene similar to $M$. incognita 14-3-3b also fell into cluster 4. Additional candidate SCN parasitism genes in cluster 4 included the pioneer gene family 30D08/16A01/21E12 (Gao et al. 2003). A gene similar to $M$. incognita calreticulin (Jaubert et al. 2005) fell into cluster 5 . The genes in this cluster did not show any significant changes between adjacent time points. Other genes in this cluster include $E N G 1$ (Gao et al. 2004; Smant et al. 1998), dorsal gland pioneer genes SB84 (Hgg-20), 32E03, and the 30D08/21E12/16A01 family.

The expression profiles of the putative SCN parasitism genes SYV42, SYV46, and SYV52 (now Eng-1) (Wang et al. 2001) previously were determined by nematode cDNA microarrays (De Boer et al. 2002b) and the overall expression profiles are consistent with our study. In addition, expression profiles of candidate parasitism genes SYS7, SYS79, SYS86, and SYV55 identified from microaspirated gland cell contents and screened by signal peptide selection in yeast (Wang et al. 2001) were profiled by De Boer and associates (2002b). All four genes were detected in our microarray study here, but are excluded from our list of SCN parasitism genes because, although they contain a signal peptide, they have never been confirmed to be expressed in the esophageal gland cells. These genes fell into clusters 3, 8, 2, and 2. SY20, SYS16, and SYV80 were not found in our list of 1,871 genes at 5\% FDR to be differentially expressed across the time points used here.

The fact that the majority of previously identified putative parasitism genes fell into three clusters with similar profiles (i.e., expression level is high at 2 dpi compared with $10 \mathrm{dpi}$ ) allows us to speculate that other members of these clusters with unknown functions are likely candidates for novel parasitism genes involved in the cyst nematode-plant interaction. However, considering the fact that transition from the motile preparasitic stage to sedentary parasitic stages also occurs during early stages of parasitism, some of the transcripts in these clusters also may be involved in that developmental transition. For example, two putative cuticular collagen genes fell into clusters 8 and 9 with the majority of putative parasitism genes. Nevertheless, our observations warrant further studies to isolate full-length clones of corresponding unknown ESTs identified in these clusters. Bioinformatic analysis of sequences for the presence of signal peptides and in situ hybridization to detect gland-specific expression likely will lead to the identification of additional novel candidate cyst nematode parasitism genes.

A comparison of the cluster analysis for differentially expressed plant and nematode genes from this study also provides novel insights into the plant-nematode interaction. For example, nematode cluster 2 is the largest cluster, with 729 genes. The genes in this cluster show significant upregulation from 2 to 5 dpi but nonsignificant changes between 5 and 10 dpi. Many of the genes in this cluster are involved in metabolism and included ribosomal proteins, ATP synthase, pyruvate dehydrogenase, alcohol dehydrogenase, and so on. Similarly, soybean-infected cluster 2, which exhibits a similar expression pattern as that of nematode cluster 2, represents 68 genes, most of them involved in plant defense responses and cell wall modification. The members of this cluster included PR proteins, GSTs, osmotin-like proteins, extensins, expansins, and germin-like proteins. Increased feeding activity and nematode body expansion within the root may be inducing plant defense and wound responses at this stage. Despite these trends, the large majority of differentially expressed nematode and soybean genes identified in this study are of unknown function. In the near future, availability of the complete soybean and nematode genome sequences with more accurate annotations will help us integrate our observations of the differential expression of both plant and nematode genes from this study.

Cross hybridization among related transcripts from a gene family, possible redundancy of transcripts on the chip, poor annotation, and limited functional genomics resources for soybean are some of the limitations that need to be considered when drawing conclusions from this study. Interestingly, this study identified only 429 differentially expressed genes (approximately $1.2 \%$ ) of the total 35,611 soybean transcripts measured, in contrast to 1,850 differentially expressed genes (approximately $24 \%$ ) of the total 7,431 nematode transcripts. This may be due to dilution of nematode responsive cells in the excised whole root tissues used in this study. We applied stringent experimental, biological, and statistical measures to make the results meaningful and reliable. Inherent biological variation in the system was reduced to a minimum by bulking samples, controlling the number of nematodes at each inoculation, and synchronizing the infection. Furthermore, we used three biological replicates for microarray analysis. The microarray results and the sequence identity for selected targets were validated by qPCR analysis. In addition, our microarray data is in agreement with results from previous studies that employed microarrays as well as those from more traditional methods of expression analysis. These comparisons provide additional verification of our results, giving a high degree of confidence in the data.

In summary, our analysis using the latest microarray technology available provides a global profile of changes in soybean and SCN gene expression during different stages of nematode parasitism. We have identified several novel genes, both in soybean and $\mathrm{SCN}$, whose expression level changes during nematode infection of roots. More detailed biological analysis of the genes identified in this study will provide a better understanding of SCN parasitism of soybean and help to identify potential targets for genetic improvement of crop plants for enhanced SCN resistance. Ultimately, identifying key biological processes involved in cyst nematode feeding-cell formation will require a combined approach of cell-type-specific isolation (Klink et al. 2005; Ramsay et al. 2004) and microarray profiling over a time course of syncytium development, and these experiments are underway.

\section{MATERIALS AND METHODS}

\section{Plant material and nematodes.}

Soybean (G. $\max ($ L.) Merr.) cv. Williams 82 was used in this study. Soybean cyst nematode $(H$. glycines Ichinohe) inbred line PA3 (HG type 0, race 3 ) was maintained by mass selection on susceptible cv. Williams 82 in greenhouse cultures. 


\section{Plant infections.}

Soybean cv. Williams 82 seed were soaked in running water for $30 \mathrm{~min}$ and surface sterilized in $10 \%$ bleach for $10 \mathrm{~min}$ followed by rinsing in running water for $30 \mathrm{~min}$. Seed were germinated for 2 days in ragdolls at $28^{\circ} \mathrm{C}$ in dark. Two-day-old seedlings with a $2-$ to $3-\mathrm{cm}$-long uniform root were inoculated with freshly hatched nematodes according to Mahalingam and associates (1998), with the following modifications. Cysts of SCN were extracted from infested soil by flotation in water and collected on a 250- $\mu \mathrm{m}$ (no. 60) sieve. Harvested cysts were crushed gently using a drill press and the eggs collected on a 25- $\mu \mathrm{m}$ (no. 500) sieve (Faghihi and Ferris 2000) and further purified on a sucrose density gradient. The eggs were sterilized in $0.02 \%$ sodium azide for 20 min and washed extensively in sterile water to remove any traces of sodium azide. Preparasitic J2s were isolated by hatching eggs in the presence of gentamycin at $1.5 \mathrm{mg} / \mathrm{ml}$ and nystatin at $0.05 \mathrm{mg} / \mathrm{ml}$ at $28^{\circ} \mathrm{C}$ on a modified Baermann pan for 2 days. Hatched juveniles were collected, rinsed with sterile water, and suspended in $0.1 \%$ sterile agarose. Then, $100 \mu \mathrm{l}$ of $0.1 \%$ agarose containing $300 \pm 25$ preparasitic $\mathbf{J} 2$ s or agarose alone (mock) was applied approximately $1 \mathrm{~cm}$ above the root tip of each soybean root which was marked with a dot using a black Sharpie pen. Infection was synchronized by washing the infected roots with water exactly $24 \mathrm{~h}$ after inoculation. Seedlings were rolled into ragdolls, placed in Hoagland's nutrient solution (1/10 dilution), and allowed to grow in a plant growth chamber at $26^{\circ} \mathrm{C}$ with a photoperiod of $16 \mathrm{~h}$ of light and $8 \mathrm{~h}$ of dark with constant aeration. Samples were collected at 2, 5, and 10 dpi. For total RNA isolation, root pieces of approximately $2 \mathrm{~cm}$ in length were excised using a scalpel at the zone of maximum infection, which could be located by the black dot on the root made with a Sharpie pen. At later time points, lateral roots were removed from excised root pieces. Excised root pieces from 30 different plants were pooled at each time point and flash frozen in liquid nitrogen. All experiments were repeated two more times for a total of three independent biological replicates.

For each time point, a random sample of 10 roots were stained to determine the number and life stage of the juveniles, as described by Dropkin and associates (1969). Briefly, roots were washed in $10 \%$ household bleach for $7 \mathrm{~min}$ and then rinsed in tap water for $20 \mathrm{~min}$. Acid fuschin stain $(1 \mathrm{ml}$ of 0.35 $\mathrm{g}$ of acid fuschin in $25 \mathrm{ml}$ of glacial acetic acid) was added to a beaker containing $50 \mathrm{ml}$ of boiling water. Roots were boiled in the solution for $1 \mathrm{~min}$, then allowed to cool at room temperature for $30 \mathrm{~min}$. Roots were rinsed in water and transferred to a beaker containing warm glycerol and incubated at $65^{\circ} \mathrm{C}$ for several hours. The stained roots were squashed between two glass slides, and juveniles were counted and photographed under a stereomicroscope. For light microscopy of feeding cells, infected root pieces were fixed in $4 \%$ paraformaldehyde and embedded in paraffin, and 5- $\mu \mathrm{m}$ sections were stained with toluidine blue stain.

\section{RNA extraction.}

Total RNA was extracted from excised whole root tissues using the RNeasy Plant mini kit (Qiagen, Valencia, CA, U.S.A.) and treated with RNase-free DNase (Qiagen) following the manufacturer's instructions. Total RNA samples were sent to the Iowa State University GeneChip microarray core facility where cDNA and cRNA preparation, fragmentation, hybridization, staining, and scanning steps were performed according to the manufacturer's recommended protocols (Affymetrix, Santa Clara, CA, U.S.A.).

Freshly hatched preparasitic J2s of SCN PA3 were collected and washed in sterile water. A $100-\mu l$ nematode pellet was flash frozen in liquid nitrogen. The pellet was ground using a 200- $\mu$ l Kontes Dual tissue grinder (Fisher Scientific, Pittsburgh, PA, U.S.A.) in the presence of PureScript cell lysis buffer (Gentra Systems, Minneapolis, MN, U.S.A.). Total RNA was extracted using the PureScript RNA isolation kit (Gentra Systems), and on-column DNase I treatment (Qiagen) was performed following the manufacturer's recommendations.

\section{Microarray chip description.}

The GeneChip soybean genome array (Affymetrix) was used in study. This oligonucleotide array contains 37,593 probesets representing 35,611 soybean transcripts, 7,530 probesets representing 7,431 soybean cyst nematode transcripts, and more than 15,800 probesets representing 15,421 Phytophthora sojae transcripts. A description of the GeneChip soybean genome array is available at the manufacturer's website.

\section{Data analysis.}

GeneChip Operating Software version 1.0 (GCOS v. 1.0) signal data were natural-log transformed and normalized by centering the log-scale median for each chip at 0 . Mixed linear model analyses using the normalized data were conducted separately for each gene. When analyzing soybean genes, each mixed linear model included fixed effects for treatments (infected or uninfected), times $(2,5$, or $10 \mathrm{dpi})$, and treatmenttime interactions, as well as random effects for replications and replication-time interactions. This is a standard mixed model for a split-plot experimental design (Kuehl, 2000) with time as the whole-plot factor and treatment as the split-plot factor. $F$ tests, resulting in $P$ values, were performed to test for the significance of each factor, to test for treatment effects separately at each time point, and to test for changes of expression across adjacent time points within each treatment. The $P$ values for each test were converted to $q$ values using the method described by Storey and Tibshirani (2003). For each test of interest, genes with q values less than 0.05 were declared statistically significant to produce a list of genes with an estimated FDR of 5\%. This gene list was further filtered by applying a cutoff of a 1.5 -fold change between mock and treatment group.

A different analysis strategy was necessary for analyzing nematode genes because there were no nematode transcripts present in mock-infected tissues. Thus, the data from the nematode genes were analyzed as a completely randomized design with time as the only factor. Analysis again was performed on a gene-by-gene basis to obtain $F$ tests for changes over time. The $P$ values from these tests were converted to $\mathrm{q}$ values, and the genes with $q$ values less than 0.05 were declared statistically significant as described for the soybean genes. The genes exhibiting more than a 1.5 -fold change between at least one of the three time combinations ( 2 to 5,5 to 10 , and 2 to $10 \mathrm{dpi}$ ) were considered biologically significant in this analysis.

The 1,850 nematode genes that exhibited significant (q value $<0.05$ and fold change $>1.5$ ) nonconstant expression across levels of dpi were separated into clusters according to expression patterns. Estimated means of adjacent levels of dpi were compared using $t$ tests. The $P$ values from these $t$ tests were used to group genes into clusters. Specifically, the genes were categorized by whether the mean expression at 2 dpi was significantly greater than, less than, or not significantly different from $5 \mathrm{dpi}$ and whether the mean expression at $5 \mathrm{dpi}$ was significantly greater than, less than, or not significantly different from 10 dpi. In each case, significance was defined by $P$ value $<0.05$. This testing for differences in expression across adjacent time points created nine clusters, including a cluster with genes that exhibited significant nonconstant expression over 
time despite nonsignificant changes from 2 to 5 dpi and from 5 to $10 \mathrm{dpi}$. Some of these genes gradually increased over the time course whereas others gradually decreased, which explains the " $X$ " shape of this small cluster.

For comparison with the nematode expression patterns, the infected and uninfected expression profiles of the 429 soybean genes with significant treatment effects were clustered using the clustering strategy employed for the nematode genes. The $\mathrm{q}$ values from the mixed-linear-model tests for changes of expression across adjacent time points within each treatment were used to place each profile into one of the nine clusters.

The nucleotide sequences of the ESTs corresponding to the differentially expressed probeset identified from microarray analysis were obtained from the Affymetrix NetAffix analysis center. Soybean EST sequences were used as a query to search for Arabidopsis orthologs in the TAIR database using WUBLAST2 search. The soybean genes were annotated based on the Arabidopsis top hit with an e value $<10^{-3}$. Nematode genes were annotated by BLASTX searches against the nonredundant protein sequence database at the National Center for Biotechnology Information. SEQtools 8.2 software was used for batch analysis of sequences. The microarray data has been deposited in the ArrayExpress database at the European Bioinformatics Institute (EBI) under accession number E-MEXP-808.

\section{qPCR analysis.}

qPCR was carried out using the Applied Biosystems 7500 Real-Time PCR system. Specific primers for each gene selected were designed using Primer Express software (Applied Biosystems, Foster City, CA, U.S.A.). For quantifications, a cDNA template prepared from the total RNA from the same biological replicates used for microarray analysis was used. qPCR was conducted in triplicate in $20 \mu \mathrm{l}$ using SYBR Green PCR master mix (Applied Biosystems). The qPCR reactions were conducted in triplicate for each biological replicate. PCR conditions used were $50^{\circ} \mathrm{C}$ for $2 \mathrm{~min}$ and $95^{\circ} \mathrm{C}$ for $10 \mathrm{~min}$, followed by 40 cycles of $95^{\circ} \mathrm{C}$ for $15 \mathrm{~s}$ and $60^{\circ} \mathrm{C}$ for $1 \mathrm{~min}$. A soybean gene encoding glutathione- $S$-transferase ( $G S T a$-accession number AF048978) was used as an internal control for soybean. This is one of seven soybean internal control genes represented on the Genechip soybean genome array. We determined by qPCR that the expression of this GST remained relatively constant across treatments compared with the other internal controls and it was chosen for validation studies. However, additional probesets for glutathione- $S$-transferase family members are printed on the chip and several of these displayed differential expression across treatments. An SCN GAPDH (accession number CA939315) was used as an internal control for SCN qPCR. Nematode GAPDH and actin were used as control genes on the GeneChip soybean genome array. The expression of GAPDH and actin was tested by qPCR and we determined that SCN GAPDH remained relatively constant across nematode developmental stages compared with actin. The RNA samples from inoculated soybean roots were used for SCN qPCR. The RNA extracted from freshly hatched preparasitic $\mathrm{J} 2 \mathrm{~s}$ also was included for comparison in qPCR analysis of SCN gene expression. The quantification of gene expression was performed using the relative $\Delta \Delta \mathrm{C}_{\mathrm{T}}$ method by comparing the data with internal control genes whose expression was determined to remain constant under different treatment conditions.

\section{ACKNOWLEDGMENTS}

This work was supported by the United States Department of Agriculture National Research Initiative Competitive Grants Program award \#2005-35604-15434 to T. J. Baum, M. G. Mitchum, and D. Nettleton. We thank R. Heinz and M. Moragudivenkata for excellent technical assistance.

\section{LITERATURE CITED}

Barthels, N., van der Lee, F. M., Klap, J., Goddijn, O. J., Karimi, M., Puzio, P., Grundler, F. M., Ohl, S. A., Lindsey, K., Robertson, L., Robertson, W. M., Van Montagu, M., Gheysen, G., and Sijmons, P. C. 1997. Regulatory sequences of Arabidopsis drive reporter gene expression in nematode feeding structures. Plant Cell 9:2119-2134.

Bird, D. M. 1996. Manipulation of host gene expression by root-knot nematodes. J Parasitol. 82:881-888.

Bird, D. M., and Koltai, H. 2000. Plant parasitic nematodes: Habitats, hormones, and horizontally acquired genes. J. Plant Growth Regul. 19:183194.

Davis, E. L., Hussey, R. S., Baum, T. J., Bakker, J., and Schots, A. 2000. Nematode parasitism genes. Annu. Rev. Phytopathol. 38:365-396.

Davis, E. L., Hussey, R. S., and Baum, T. J. 2004. Getting to the roots of parasitism by nematodes. Trends Parasitol. 20:134-141.

Davis, E. L., and Mitchum, M. G. 2005. Nematodes. Sophisticated parasites of legumes. Plant Physiol. 137:1182-1188.

De Boer, J. M., Yan, Y. T., Wang, X. H., Smant, G., Hussey, R. S., Davis, E. L., and Baum, T. J. 1999. Developmental expression of secretory beta-1,4-endoglucanases in the subventral esophageal glands of Heterodera glycines. Mol. Plant-Microbe Interact. 12:663-669.

De Boer, J. M., McDermott, J. P., Davis, E. L., Hussey, R. S., Popeijus, H., Smant, G., and Baum, T. J. 2002a. Cloning of a putative pectate lyase gene expressed in the subventral esophageal glands of Heterodera glycines. J. Nematol. 34:9-11.

De Boer, J. M., McDermott, J. P., Wang, X., Maier, T, Qui, F., Hussey, R. S., Davis, E. L., and Baum, T. J. 2002b. The use of DNA microarrays for the developmental expression analysis of cDNAs from the oesophageal gland cell region of Heterodera glycines. Mol. Plant Pathol. 3:261-270.

Dropkin, V. H., Helgeson, J. P., and Upper, C. D. 1969. The hypersensitivity reaction of tomato resistant to Meloidogyne incognita: Reversal by cytokinins. J. Nematol. 1:55-61.

Edens, R. M., Anand, S. C., and Bolla, R. I. 1995. Enzymes of the phenylpropanoid pathway in soybean infected with Meloidogyne incognita or Heterodera glycines. J. Nematol. 27:292-303.

Endo, B. Y. 1986. Pages 133-146 in: Histology and Ultrastructural Modification Induced by Cyst Nematodes F. Lamberti and C. E. Taylor, eds. Plenum Press, New York.

Faghihi, J., and Ferris J. M. 2000. An efficient new device to release eggs from Heterodera glycines. J. Nematol. 32:411-413.

Favery, B., Lecomte, P., Gil, N., Bechtold, N., Bouchez, D., Dalmasso, A., and Abad, P. 1998. RPE, a plant gene involved in early developmental steps of nematode feeding cells. EMBO (Eur. Mol. Biol. Organ.) J. 17:6799-6811.

Gao, B. L., Allen, R., Maier, T., Davis, E. L., Baum, T. J., and Hussey R. S. 2001. Identification of putative parasitism genes expressed in the esophageal gland cells of the soybean cyst nematode Heterodera glycines. Mol. Plant-Microbe Interact. 14:1247-1254.

Gao, B. L., Allen, R., Maier, T., McDermott, J. P., Davis, E. L., Baum T. J., and Hussey, R. S. 2002. Characterisation and developmental expression of a chitinase gene in Heterodera glycines. Int. J. Parasitol. 32:1293-1300.

Gao, B. L., Allen, R., Maier, T., Davis, E. L., Baum T. J., and Hussey, R. S. 2003. The parasitome of the phytonematode Heterodera glycines. Mol. Plant-Microbe Interact. 16:720-726.

Gao, B. L., Allen, R., Davis, E. L., Baum, T. J., and Hussey, R. S. 2004. Developmental expression and biochemical properties of a beta-1,4endoglucanase family in the soybean cyst nematode, Heterodera glycines. Mol. Plant Pathol. 5:93-104.

Gheysen, G., and Fenoll C. 2002. Gene expression in nematode feeding sites. Annu. Rev. Phytopathol. 40:191-219.

Goddijn, O. J. M., Lindsey, K., Vanderlee, F. M., Klap, J. C., and Sijmons, P. C. 1993. Differential gene expression in nematode-induced feeding structures of transgenic plants harboring promoter GusA fusion constructs. Plant J. 4:863-873.

Goellner, M., Smant, G., De Boer, J. M., Baum, T. J. and Davis, E. L. 2000. Isolation of beta-1,4-endoglucanase genes from Globodera tabacum and their expression during parasitism. J. Nematol. 32:154-165.

Goellner, M., Wang, X. H., and Davis, E. L. 2001. Endo-beta-1,4-glucanase expression in compatible plant-nematode interactions. Plant Cell 13:2241-2255.

Gopalan, S., Wei, W., and He, S. Y. 1996. hrp gene-dependent induction of hin1: A plant gene activated rapidly by both harpins and the avrPto gene-mediated signal. Plant J. 10:591-600.

Goverse, A., Bieshieuvel, J., Wijers, G.-J., Gommers, F. J., Bakker, J., Schots, A., and Helder, J. 1998. In planta monitoring of the activity of two constitutive promoters, CaMV $35 \mathrm{~S}$ and TR2', in developing feeding cells induced by Globodera rostochiensis using green fluorescent protein in combination with confocal laser scanning microscopy. Physiol. Mol. Plant Pathol. 52:275-284. 
Goverse, A., Overmars, H., Engelbertink, J., Schots, A., Bakker, J., and Helder, J. 2000. Both induction and morphogenesis of cyst nematode feeding cells are mediated by auxin. Mol. Plant-Microbe Interact. 13:1121-1129.

Gurr, S. J., McPherson, M. J., Scollan, C., Atkinson, H. J., and Bowles, D. J. 1991. Gene-expression in nematode-infected plant roots. Mol. Gen. Genet. 226:361-366.

Hermsmeier, D., Mazarei, M., and Baum, T. J. 1998. Differential display analysis of the early compatible interaction between soybean and the soybean cyst nematode. Mol. Plant-Microbe Interact. 11:1258-1263.

Hermsmeier, D., Hart, J. K., Byzova, M., Rodermel, S. R., and Baum, T. J. 2000. Changes in mRNA abundance within Heterodera schachtii-infected roots of Arabidopsis thaliana. Mol. Plant-Microbe Interact. 13:309-315.

Huang, G. Z., Gao, B. L., Maier, T., Allen, R., Davis, E. L., Baum, T. J., and Hussey, R. S. 2003. A profile of putative parasitism genes expressed in the esophageal gland cells of the root-knot nematode Meloidogyne incognita. Mol. Plant-Microbe Interact. 16:376-381.

Hussey R. S., and Grundler, F. M. 1998. Nematode parasitism of plants. Pages 213-243 in: Physiology and Biochemistry of Free-Living and Plant-Parasitic Nematodes. R. N. Perry and J. Wright, eds. CAB International Press, Oxford.

Hussey, R. S., Davis, E. L., and Baum, T. J. 2002. Secrets in secretions: Genes that control nematode parasitism in plants. Braz. J. Plant Physiol. 14:183-194

Hutangura, P., Mathesius, U., Jones, M. G. K., and Rolfe, B. G. 1999. Auxin induction is a trigger for root gall formation caused by root-knot nematodes in white clover and is associated with the activation of the flavonoid pathway. Aust. J. Plant Physiol. 26:221-231

Jammes, F. Lecomte, P., de Almeida-Engler, J., Bitton, F., MartinMagniette, M., Renou, J. P., Abad, P., and Favery, B. 2005. Genomewide expression profiling of the host response to root-knot nematode infection in Arabidopsis. Plant J. 44:447-458.

Jaubert, S., Laffaire, J. B., Ledger, T. N., Escoubas, P., Amri, E. Z., Abad, P., and Rosso, M. N. 2004. Comparative analysis of two 14-3-3 homologues and their expression pattern in the root-knot nematode Meloidogyne incognita. Int. J. Parasitol. 34:873-880.

Jaubert, S., Milac, A. L., Petrescu, A. J., de Almelda-Engler, J., Abad, P., and Rosso, M. N. 2005. In planta secretion of a calreticulin by migratory and sedentary stages of root-knot nematode. Mol. Plant-Microbe Interact. 18:1277-1284.

Keen, N. T. 1992. The molecular biology of disease resistance. Plant Mol. Biol. 19:109-122.

Khan, R., Alkharouf, N., Beard, H., MacDonald, M., Chouikha, I., Meyer, S., Grefenstette, J., Knap, H., and Matthews, B. 2004. Microarray analysis of gene expression in soybean roots susceptible to the soybean cyst nematode two days post invasion. J. Nematol. 36:241-248.

Kim, C. Y., Lee, S. H., Park, H. C., Bae, C. G., Cheong, Y. H., Choi, Y. J., Han, C., Lee, S. Y., Lim, C. O., and Cho, M. J. 2000. Identification of rice blast fungal elicitor-responsive genes by differential display analysis. Mol. Plant-Microbe Interact. 13:470-474.

Klink, V. P., Alkharouf, N., MacDonald, M., and Matthews, B. 2005. Laser capture microdissection (LCM) and expression analyses of Glycine max (soybean) syncytium containing root regions formed by the plant pathogen Heterodera glycines (soybean cyst nematode). Plant Mol. Biol. 59:965-979.

Kuehl, R. O. 2000. Design of Experiments: Statistical Principles of Research Design and Analysis, 2nd ed. Duxbury Press, Pacific Grove, CA, U.S.A.

Lambert, K. N., Allen, K. D., and Sussex, I. M. 1999. Cloning and characterization of an esophageal-gland-specific chorismate mutase from the phytoparasitic nematode Meloidogyne javanica. Mol. Plant-Microbe Interact. 18:593-601

Le Quere, A., Wright, D. P., Soderstrom, B., Tunlid, A., and Johanson, T. 2005. Global patterns of gene regulation associated with development of ectomycorrhiza between Birch (Betula pendula Roth.) and Paxillus involutus (Batsch) Fr. Mol. Plant-Microbe Interact. 18:659-673.

Mahalingam, R., Knap, H. T., and Levis, S. A. 1998. Inoculation method for studying early responses of Glycine max to Heterodera glycines. J. Nematol. 30:237-240.
Mazarei, M., Lennon, K. A., Puthoff, D. P., Rodermel, S. R., and Baum, T. J. 2003. Expression of an Arabidopsis phosphoglycerate mutase homologue is localized to apical meristems, regulated by hormones, and induced by sedentary plant-parasitic nematodes. Plant Mol. Biol. 53:513-530.

Mazarei, M., Lennon, K. A., Puthoff, D. P., Rodermel, S. R., and Baum, T. J. 2004. Homologous soybean and Arabidopsis genes share responsiveness to cyst nematode infection. Mol. Plant Pathol. 5:409-423.

Moy, P., Qutob, D., Chapman, B. P., Atkinson, I., and Gijzen, M. 2004. Patterns of gene expression upon infection of soybean plants by Phytophthora sojae. Mol. Plant-Microbe Interact. 17:1051-1062

Niebel, A., de Almeida-Engler. J., Hemerly, A., Ferreira, P., Inze, D., Van Montagu, M., and Gheysen, G. 1996. Induction of $c d c 2 a$ and $c y c 1 A t$ expression in Arabidopsis thaliana during early phases of nematodeinduced feeding cell formation. Plant J. 10:1037-1043.

Niebel, A., de Almeida-Engler. J., Tire, C., Engler, G., Van Montagu, M., and Gheysen, G. 1993. Induction patterns of an extensin gene in tobacco upon nematode infection. Plant Cell 5:1697-1710.

Puthoff, D. P., Nettleton, D., Rodermel, S. R., and Baum, T. J. 2003. Arabidopsis gene expression changes during cyst nematode parasitism revealed by statistical analyses of microarray expression profiles. Plant J. 33:911-921.

Puzio, P. S., Lausen, J., Heinen, P., and Grundler, F. M. W. 2000. Promoter analysis of pyk20, a gene from Arabidopsis thaliana. Plant Sci. 157:245-255.

Qin, L., Kudla, U., Roze, E. H. A., Goverse, A., Popeijus, H., Nieuwland, J., Overmars, H., Jones, J. T., Schots, A., Smant, G., Bakker, J., and Helder, J. 2004. Plant degradation: A nematode expansin acting on plants. Nature 427:30-30.

Ramsay, K., Wang, Z. H., and Jones, M. G. K. 2004. Using laser capture microdissection to study gene expression in early stages of giant cells induced by root-knot nematodes. Mol. Plant Pathol. 5:587-592.

Smant, G., Stokkermans, J. P. W. G., Yan, Y. T., de Boer, J. M., Baum, T. J., Wang, X. H., Hussey, R. S., Gommers, F. J., Henrissat, B., Davis, E. L., Helder, J., Schots, A., and Bakker, J. 1998. Endogenous cellulases in animals: Isolation of beta-1,4-endoglucanase genes from two species of plant-parasitic cyst nematodes. Proc. Natl. Acad. Sci. U.S.A. 95:49064911.

Storey, J. D., and Tibshirani, R. 2003. Statistical significance for genomewide studies. Proc. Natl. Acad. Sci. U.S.A. 100:9440-9445.

Tian, Z. D., Liu, J., Xie, C. H., and Song, B. T. 2003. Cloning of potato POTHR-1 gene and its expression in response to infection by Phytophthora infestans and other abiotic stimuli. Acta Bot. Sin. 45:959965.

Vanneste, S., De Rybel, B., Beemster, G. T. S., Ljung, K., De Smet, I., Van Isterdael, G., Naudts, M., Iida, R., Gruissem, W., Tasaka, M., Inzé, D. Fukaki, H., and Beeckman, T. 2005. Cell cycle progression in the pericycle is not sufficient for SOLITARY ROOT/IAA14-mediated lateral root initiation in Arabidopsis thaliana. Plant Cell 17:3035-3050.

Vercauteren, I., Van Der Schueren, E., Van Montagu, M., and Gheysen, G. 2001. Arabidopsis thaliana genes expressed in the early compatible interaction with root-knot nematodes. Mol. Plant-Microbe Interact. 14:288-299.

Wang, X. H., Allen, R., Ding, X. F., Goellner, M., Maier, T., de Boer, J. M., Baum, T. J., Hussey, R. S., and Davis, E. L. 2001. Signal peptideselection of cDNA cloned directly from the esophageal gland cells of the soybean cyst nematode Heterodera glycines. Mol. Plant-Microbe Interact. 14:536-544.

Williamson, V. M., and Gleason, C. A. 2003. Plant-nematode interactions. Curr. Opin. Plant Biol. 6:327-333.

Wrather, J. A., Stienstra, W. C., and Koenning, S. R. 2001. Soybean disease loss estimates for the United States from 1996-1998. Can. J. Plant Pathol. 23:122-131.

\section{AUTHOR-RECOMMENDED INTERNET RESOURCES}

ArrayExpress database: www.ebi.ac.uk/arrayexpress/

GeneChip: www.affymetrix.com/products/arrays/specific/soybean.affx NetAffix analysis center: www.affymetrix.com/analysis/netaffx/index.affx SEQtools 8.2: www.seqtools.dk 